\title{
From Newton's method to exotic basins Part I: The parameter space
}

\author{
by
}

\author{
Krzysztof Bar ań s ki (Warszawa)
}

\begin{abstract}
This is the first part of the work studying the family $\mathfrak{F}$ of all rational maps of degree three with two superattracting fixed points. We determine the topological type of the moduli space of $\mathfrak{F}$ and give a detailed study of the subfamily $\mathcal{F}_{2}$ consisting of maps with a critical point which is periodic of period 2. In particular, we describe a parabolic bifurcation in $\mathcal{F}_{2}$ from Newton maps to maps with so-called exotic basins.
\end{abstract}

1. Introduction. In this paper we study the family $\mathfrak{F}$ of all rational mappings $f$ of degree three with two superattracting fixed points $c_{1}, c_{2}$. Up to conformal conjugation, it can be written as the following two-parameter family $\mathcal{F}=\left\{f_{a, b}\right\}$ :

$$
f_{a, b}(z)=a z^{2} \frac{b z+1-2 b}{(2-b) z-1}, \quad a \in \mathbb{C} \backslash\{0\}, b \in \mathbb{C} \backslash\{0,1\},
$$

where 0 and $\infty$ are supersinks and 1 is a critical point. In particular, $\mathcal{F}$ contains the families of cubic Newton maps and cubic polynomials with a supersink.

The study of the spaces of rational maps of degree $d$ is far from being developed. Except some works describing general topological properties of these spaces (e.g. [Mi2] in the case of $d=2$ and [Se]), the greatest progress was made in the quadratic case, due to the works by M. Rees, which aim at a complete description of the space of quadratic rational maps (see [Re1], $[\operatorname{Re} 2])$. However, this encounters serious difficulties and requires a complicated combinatorial description. The usual procedure is to study complex slices in the parameter space obtained by fixing the behaviour of some critical points. A well-known example is the family of Newton maps, studied in e.g. $[\mathrm{HP}],[\mathrm{P} 1],[\mathrm{Sh}]$.

1991 Mathematics Subject Classification: Primary 58F23.

Research supported by Polish KBN Grants No 2 P301 01307 and 2 P03A 02512. 
This work contributes to the study of the space of cubic rational maps. We examine the two-parameter family $\mathcal{F}$ consisting of cubic rational maps with two supersinks. The work was motivated by the paper [P1] by Feliks Przytycki. He introduced the notion of "exotic" basins for rational maps as non-simply connected completely invariant basins of attraction containing $k$ critical points with $k$ less than the degree of the map. He also described a one-parameter family of maps joining a cubic Newton map to maps with exotic basins and asked if Mandelbrot-like sets in the parameter plane of cubic Newton maps (see [CGS], [DH2]) move continuously under the change of parameters to "exotic" Mandelbrot-like sets.

In this work we answer this question in the affirmative. We also give a description of the space $\mathcal{F}$ and a detailed study of a one-parameter subfamily $\mathcal{F}_{2} \subset \mathcal{F}$ consisting of maps with a periodic critical point of period two. The work is divided into two papers. In the first one we describe the parameter space of $\mathcal{F}$ and $\mathcal{F}_{2}$ and prove the existence of a parabolic bifurcation in $\mathcal{F}_{2}$ from a Newton map via a parabolic map to maps with exotic or non-exotic basins. The second paper generalizes this result to the case of a parabolic bifurcation of Mandelbrot-like sets in $\mathcal{F}$.

Let us summarize the content of the paper. Section 2 presents preliminary facts about the families $\mathfrak{F}$ and $\mathcal{F}$. In Section 3 we determine the topology of the moduli space $\mathcal{M}(\mathfrak{F})$ (i.e. the space of holomorphic conjugacy classes of maps from $\mathfrak{F}) . \mathcal{M}(\mathfrak{F})$ is an orbifold which is the quotient space of $\mathfrak{F}$ under the action of the Möbius group. We prove that $\mathcal{M}(\mathfrak{F})$ is an algebraic set in $\mathbb{C}^{3}$ of complex dimension two, with singularities at points corresponding to Newton maps. In particular, $\mathcal{M}(\mathfrak{F})$ is not a manifold. This is a difference compared to the case of the moduli space of quadratic rational maps and of cubic polynomials. (Both these spaces are homeomorphic to $\mathbb{C}^{2}$ - see [Mi2].)

Since maps from $\mathfrak{F}$ have two "free" critical points, by fixing the behaviour of one of them, one can obtain various one-parameter subfamilies of maps of degree three. One of the simplest cases is when we require that one of the two critical points is double-critical. This gives (up to conformal conjugation) the family of cubic polynomials with a critical fixed point. (For the description of the space of cubic polynomials we refer to [BH].) Another condition is making one of the "free" critical points fixed. This leads to the well-known family of cubic Newton maps (see [CGS], [He], [Ta], [Ro]). In Section 4 we study a one-parameter family $\mathcal{F}_{2} \subset \mathcal{F}$ defined by another simple condition. We require that the critical point 1 is periodic with period two. $\mathcal{F}_{2}$ is parameterized by a parameter $\lambda \in \Lambda$, with $\Lambda$ equal to $\widehat{\mathbb{C}}$ without a finite number of singular parameters where $f_{\lambda}$ degenerates to a map of lower degree. We study the behaviour of the fourth "free" critical point $u_{\lambda}$. 
Subsection 4.1 contains basic definitions and describes the general properties of $\mathcal{F}_{2}$. Subsection 4.2 is devoted to the study of the plane $\Lambda$ near singular parameters. We describe some similarities between neighbourhoods of some singular parameters and neighbourhoods of some points of the Julia sets of quadratic polynomials.

In Subsection 4.3 we prove a classification theorem for the hyperbolic components in $\Lambda$ (Theorem 4.9). We study here the components of the set of parameters for which $u_{\lambda}$ is in the immediate basin of the supersinks 0 or $\infty$. Such components can be "exotic" (when the immediate basin is exotic) or "regular" (in the other case). We show that the regular components are topological discs, and the exotic ones are punctured discs with a singular parameter in the "centre".

Subsection 4.4 presents some facts about the combinatorics of cubic Newton maps, most of them coming from $[\mathrm{He}]$ and $[\mathrm{Ta}]$.

Subsection 4.5 describes a parabolic bifurcation from a Newton map in $\mathcal{F}_{2}$ to maps with exotic or non-exotic immediate basins of a supersink (Theorem 4.20). There exists a curve on the plane $\Lambda$ joining a Newton map $N \in \mathcal{F}_{2}$ to a parabolic map $P$ with a fixed point of multiplier 1 . Then using results from [DH1] we prove the existence of two curves starting at $P$, one contained in an exotic component and the other in a regular one. In fact these curves are internal rays in these components.

In the second part of the work we will prove the existence of a parabolic bifurcation of Mandelbrot-like sets in $\mathcal{F}$. Starting from a Mandelbrot-like set in cubic Newton maps (see [DH2]) and continuously changing parameters $(a, b)$ we obtain a parabolic Mandelbrot-like set contained in the family of maps with a fixed point of multiplier 1 . Then it bifurcates to two paths of Mandelbrot-like sets, an exotic and a regular one. The regular path ends at the Mandelbrot-like set in the space of cubic polynomials (cf. $[\mathrm{BH}]$ ). Note that Theorem 4.20 describes the bifurcation of the "centre" of the main cardioid in such Mandelbrot-like sets.

Acknowledgments. This work is a part of the author's Ph.D. thesis, written at Warsaw University. The author wishes to express his special thanks to Prof. Feliks Przytycki, his thesis advisor, for his kind help and a great number of comments and suggestions he provided during the preparation of the paper.

Notation. We denote by $\widehat{\mathbb{C}}$ the Riemann sphere $\mathbb{C} \cup\{\infty\}$. $\mathbb{D}$ denotes the open unit disc $\{z \in \mathbb{C}:|z|<1\}, \mathbb{D}_{r}=\{z \in \mathbb{C}:|z|<r\}$ and $\mathbb{D}_{r}(x)=\{z \in$ $\mathbb{C}:|z-x|<r\}$. By a topological disc we mean a set homeomorphic to $\mathbb{D}$. The closure, interior and boundary of a set $A$ are denoted respectively by $\operatorname{cl} A$, int $A$ and $\partial A$. We write $J(f)$ for the Julia set of a rational map $f$. If $s$ is an attracting periodic point for a map $f$, then we write $B(s)$ for the 
immediate basin of attraction to $s$, i.e. the component of the entire basin of $s$ containing $s$. We use the symbols $f(z)=\mathcal{O}(g(z)), f(z)=o(g(z))$ in the usual sense.

2. Preliminaries. Recall that $\mathfrak{F}$ denotes the family of all cubic rational maps with two superattracting fixed points $c_{1}, c_{2}$. First we find a suitable parameterization of $\mathfrak{F}$. Take a map from $\mathfrak{F}$ and apply a conformal change of coordinates such that $c_{1}=0, c_{2}=\infty$. Then $f$ has the following form:

$$
f(z)=z^{2} \frac{z+\alpha}{\beta z+\gamma}, \quad \alpha, \beta, \gamma \in \mathbb{C}, \gamma \neq 0, \gamma-\alpha \beta \neq 0 .
$$

Consider first the case when $f$ has no critical points except 0 and $\infty$. This condition is equivalent to $\alpha=\beta=0$. Then $f(z)=z^{3} / \gamma$ and the map is conformally conjugate to $z^{3}$. (Thus, the maps with exactly two critical points are represented by one point in the moduli space.) Neglecting this trivial case, we assume from now on that $f$ has at least three critical points. Applying a suitable conformal change of coordinates to a map of the form (1) we can assume $f$ has two supersinks $0, \infty$, and the third critical point is $c_{3}=1$. This means $\beta(\alpha+2)+\gamma(2 \alpha+3)=0$. Note that since $\gamma \neq 0$, this implies $\alpha+2 \neq 0$. Hence, we can write $f$ in the form

$$
f(z)=-\frac{\alpha+2}{\gamma} z^{2} \frac{z+\alpha}{(2 \alpha+3) z-\alpha-2} .
$$

Introducing new parameters $a=-(\alpha+2) / \gamma$ and $b=1 /(\alpha+2)$ we obtain

$$
f_{a, b}(z)=a z^{2} \frac{b z+1-2 b}{(2-b) z-1}, \quad a \in \mathbb{C} \backslash\{0\}, b \in \mathbb{C} \backslash\{0,1\} .
$$

Definition 2.1. Let $\mathcal{F}=\left\{f_{a, b}\right\}_{a, b}$ be the family of all the maps of the form (2).

This family will be studied throughout the paper. One can easily check that:

the critical points of $f_{a, b}$ are $0, \infty, 1, u=\frac{2 b-1}{b(2-b)}$;

$$
f_{a, b}(0)=0, f_{a, b}(\infty)=\infty, f_{a, b}(1)=a, f_{a, b}(u)=\frac{a(2 b-1)^{3}}{b(2-b)^{3}} .
$$

Consider singular values of the parameters $a, b$. When $a=0$ (resp. $\infty$ ), the map $f_{a, b}$ degenerates to the constant 0 (resp. $\infty$ ). Assume $a \neq 0, \infty$. For $b=\infty, f_{a, b}$ is the quadratic polynomial $a z(2-z)$ and 0 is not a critical point. Similarly, for $b=0, f_{a, b}$ becomes the quadratic rational map $a \frac{z^{2}}{2 z-1}$ and $\infty$ is not a critical point. Finally, for $b=1, f_{a, b}(z)=a z^{2}$, where 1 is not a critical point. Moreover, we have 
Lemma 2.2. Let $a_{0} \neq 0, \infty$. Then:

- If $(a, b) \rightarrow\left(a_{0}, \infty\right)$, then $f_{a, b}(z) \rightarrow a_{0} z(2-z)$ uniformly in the spherical metric on every set $U \subset \widehat{\mathbb{C}}$ such that $0 \notin \operatorname{cl} U$.

- If $(a, b) \rightarrow\left(a_{0}, 0\right)$, then $f_{a, b}(z) \rightarrow a_{0} \frac{z^{2}}{2 z-1}$ uniformly in the spherical metric on every set $U \subset \widehat{\mathbb{C}}$ such that $\infty \notin \mathrm{cl} U$.

- If $(a, b) \rightarrow\left(a_{0}, 1\right)$, then $f_{a, b}(z) \rightarrow a_{0} z^{2}$ uniformly in the spherical metric on every set $U \subset \widehat{\mathbb{C}}$ such that $1 \notin \operatorname{cl} U$.

Proof. Let $(a, b) \rightarrow\left(a_{0}, \infty\right)$. Since $a z(2-z) \rightarrow a_{0} z(2-z)$ uniformly in the spherical metric on $\widehat{\mathbb{C}}$, it is sufficient to estimate the spherical distance between $f_{a, b}(z)$ and $a z(2-z)$. By $(2)$,

$$
f_{a, b}(z)-a z(2-z)=2 a \frac{z(z-1)^{2}}{(2-b) z-1} .
$$

By assumption, there exists $\delta>0$ such that $|z|>\delta$ for every $z \in U$. Suppose $|z|<R$ for a large fixed $R>0$. Then there exists a constant $c$ independent of $z \in U$ and of $a$ near $a_{0}$ such that

$$
\left|f_{a, b}(z)-a z(2-z)\right|<c \frac{R^{3}}{\delta b}
$$

which tends to 0 as $b \rightarrow \infty$. Suppose now $|z| \geq R$. Then

$$
\frac{1}{f_{a, b}(z)}-\frac{1}{a z(2-z)}=\frac{2(z-1)^{2}}{a z^{2}(z-2)(b(z-2)+1)}<\frac{c^{\prime}}{R^{2} b}
$$

for a constant $c^{\prime}$ independent of $z \in U$ and of $a$ near $a_{0}$. This implies that $f_{a, b}(z)$ tends to $a_{0} z(2-z)$ uniformly in the spherical metric on $U$. The other cases can be proved in the same way.

REMARK. Note that for $b=2, f_{a, b}$ is the polynomial $a z^{2}(3-2 z)$.

3. Orbifold structure of $\mathcal{M}(\mathfrak{F})$. In this section we describe the moduli space of $\mathfrak{F}$, which we denote by $\mathcal{M}(\mathfrak{F})$. By "moduli space" we mean the space of all holomorphic conjugacy classes of maps from $\mathfrak{F}$. As shown in Section 2, $\mathcal{M}(\mathfrak{F})$ is the union of the moduli space $\mathcal{M}(\mathcal{F})$ and one point corresponding to the maps conjugate to $z \mapsto z^{3}$. Hence we now study the space $\mathcal{M}(\mathcal{F})$.

We identify $\mathcal{M}(\mathcal{F})$ with the quotient space of

$$
\mathcal{F} \simeq\{(a, b): a \in \mathbb{C} \backslash\{0\}, b \in \mathbb{C} \backslash\{0,1\}\}
$$

under the action of a suitable group of Möbius maps. We will determine the orbifold structure of $\mathcal{M}(\mathcal{F})$, i.e. we will find a holomorphic mapping $H=H(a, b)$ with values in $\mathbb{C}^{3}$ such that $H\left(a_{1}, b_{1}\right)=H\left(a_{2}, b_{2}\right)$ if and only if $\left(a_{1}, b_{1}\right)$ and $\left(a_{2}, b_{2}\right)$ are in the same orbit of the action of the group. In particular, this gives a homeomorphism between $\mathcal{M}(\mathcal{F})$ (with the usual quotient topology) and $X \backslash\{v\}$, where $X \subset \mathbb{C}^{3}$ is an algebraic set of complex 
dimension two. The point $v$ corresponds to the unique point of $\mathcal{M}(\mathfrak{F}) \backslash \mathcal{M}(\mathcal{F})$. The set $X$ is not a manifold, since it has singularities at points corresponding to Newton maps in $\mathcal{F}$.

Definition 3.1. For $\zeta=0,1, \infty$ denote by $\mathcal{D}_{\zeta}$ the family of all maps from $\mathcal{F}$ for which $\zeta$ is a double critical point. Let $\mathcal{D}=\mathcal{D}_{0} \cup \mathcal{D}_{1} \cup \mathcal{D}_{\infty}$. Denote also by $\mathcal{N}_{(0,1, \infty)}\left(\right.$ resp. $\left.\mathcal{N}_{(0, u, \infty)}\right)$ the family of all Newton maps in $\mathcal{F}$ with three supersinks $0,1, \infty$ (resp. $0, u, \infty$ ). By a Newton map we mean here a map of degree three with three supersinks. Such a map is conformally conjugate to Newton's method for a cubic polynomial with three simple roots. Recall that Newton's method for a polynomial $p$ is defined by

$$
N_{p}(z)=z-\frac{p(z)}{p^{\prime}(z)} .
$$

Set also $\mathcal{N}=\mathcal{N}_{(0,1, \infty)} \cup \mathcal{N}_{(0, u, \infty)}$. Note that in the definition of $\mathcal{N}_{(0, u, \infty)}$ we exclude the case $u=0, \infty$. Note also that $\mathcal{N} \cap \mathcal{D} \neq \emptyset$, because a simple root of a polynomial can be a root of its second derivative and then the Newton method has a double critical point.

By (3), we have

$$
\begin{aligned}
& \mathcal{D}_{0} \simeq\{(a, b): a \in \mathbb{C} \backslash\{0\}, b=1 / 2\}, \\
& \mathcal{D}_{\infty} \simeq\{(a, b): a \in \mathbb{C} \backslash\{0\}, b=2\}, \\
& \mathcal{D}_{1} \simeq\{(a, b): a \in \mathbb{C} \backslash\{0\}, b=-1\}, \\
& \mathcal{N}_{(0,1, \infty)} \simeq\{(a, b): a=1, b \in \mathbb{C} \backslash\{0,1\}\}, \\
& \mathcal{N}_{(0, u, \infty)} \simeq\left\{(a, b): a=\left(\frac{b-2}{2 b-1}\right)^{2}, b \in \mathbb{C} \backslash\{0,1 / 2,1,2\}\right\} .
\end{aligned}
$$

The following lemma describes the action of Möbius maps on $\mathcal{F}$.

LEMMA 3.2. $\mathcal{F}$ splits into three disjoint sets invariant under the action of Möbius maps:

$$
\mathcal{F}=\mathcal{F}^{(1)} \cup \mathcal{F}^{(2)} \cup \mathcal{F}^{(3)},
$$

where $\mathcal{F}^{(1)}=\mathcal{F} \backslash\left(\mathcal{D}_{0} \cup \mathcal{D}_{\infty} \cup \mathcal{N}\right)$ is composed of maps with exactly two simple supersinks, $\mathcal{F}^{(2)}=\left(\mathcal{D}_{0} \cup \mathcal{D}_{\infty}\right) \backslash \mathcal{N}$ consists of maps with exactly two supersinks, a simple one and a double one, and $\mathcal{F}^{(3)}=\mathcal{N}$ is the family of maps with three supersinks (Newton maps). Moreover,

$$
\begin{aligned}
& \mathcal{M}\left(\mathcal{F}^{(1)}\right) \simeq \mathcal{F}^{(1)} /\left\langle g_{1}, g_{2}\right\rangle, \\
& \mathcal{M}\left(\mathcal{F}^{(2)}\right) \simeq \mathcal{F}^{(2)} /\left\langle g_{1}\right\rangle, \\
& \mathcal{M}\left(\mathcal{N}_{(0,1, \infty)}\right) \simeq \mathcal{N}_{(0,1, \infty)} /\left\langle g_{1}, g_{3}\right\rangle, \\
& \mathcal{N}_{(0, u, \infty)} \simeq{ }_{g_{2}} \mathcal{N}_{(0,1, \infty)} \backslash\left(\mathcal{D}_{0} \cup \mathcal{D}_{\infty}\right),
\end{aligned}
$$


where

$$
\begin{aligned}
g_{1}(a, b) & =\left(\frac{1}{a}, \frac{1}{b}\right), & f_{g_{1}(a, b)} & =h_{1}^{-1} \circ f_{a, b} \circ h_{1}, h_{1}(z)=\frac{1}{z}, \\
g_{2}(a, b) & =\left(\frac{1}{a}\left(\frac{b-2}{2 b-1}\right)^{2}, b\right), f_{g_{2}(a, b)} & =h_{2}^{-1} \circ f_{a, b} \circ h_{2}, h_{2}(z) & =\frac{2 b-1}{b(2-b)} \cdot \frac{1}{z}, \\
g_{3}(1, b) & =\left(1, \frac{b}{b-1}\right), & f_{g_{3}(1, b)} & =h_{3}^{-1} \circ f_{1, b} \circ h_{3}, h_{3}(z)=1-z .
\end{aligned}
$$

Proof. Let $h$ be a Möbius map conjugating two maps from $\mathcal{F}^{(1)}$. Then $h(\{0, \infty\})=\{0, \infty\}$ and $h(\{1, u\})=\{1, u\}$. Composing (if necessary) $h$ with $h_{2}$ we get $h(1)=1$. If $h(0) \neq 0$, compose $h$ with $h_{1}$. Then $h$ fixes $0,1, \infty$ so it is the identity.

If $h$ conjugates two maps from $\mathcal{F}^{(2)}$, then $h(1)=1$ and $h(\{0, \infty\})=$ $\{0, \infty\}$ so after composing $h$ (if necessary) with $h_{1}$ we have $h=$ id.

Similarly, if $h$ conjugates two maps with supersinks $0,1, \infty$, then by composing it (if necessary) with $h_{1}$ and $h_{3}$ we get the identity.

Finally, it is clear that $g_{2}$ identifies $\mathcal{N}_{(0, u, \infty)}$ with $\mathcal{N}_{(0,1, \infty)} \backslash\left(\mathcal{D}_{0} \cup \mathcal{D}_{\infty}\right)$.

Note that $g_{1}$ acts on the entire space $\mathcal{F}, g_{2}$ acts on $\mathcal{F} \backslash\left(\mathcal{D}_{0} \cup \mathcal{D}_{\infty}\right)$ and $g_{3}$ acts on $\mathcal{N}_{(0,1, \infty)}$. With this convention, we obtain

Corollary 3.3. $\mathcal{M}(\mathcal{F}) \simeq \mathcal{F} /\left\langle g_{1}, g_{2}, g_{3}\right\rangle$.

REMARK. Note that on $\mathcal{F} \backslash\left(\mathcal{D}_{0} \cup \mathcal{D}_{\infty}\right)$ we have $g_{1}^{2}=g_{2}^{2}=$ id and

$$
\left\langle g_{1}, g_{2}\right\rangle \cong \mathbb{Z}_{2} \otimes \mathbb{Z}_{2} \text {. }
$$

In fact, after the change of parameters $\mu=a \frac{2 b-1}{b-2}, \nu=b$ we get

$$
g_{1}(\mu, \nu)=\left(\frac{1}{\mu}, \frac{1}{\nu}\right), \quad g_{2}(\mu, \nu)=\left(\frac{1}{\mu}, \nu\right) .
$$

Furthermore, on $\mathcal{N}_{(0,1, \infty)}$ we have $g_{3}^{2}=$ id and

$$
\left\langle g_{1}, g_{3}\right\rangle \cong S_{3} \text {. }
$$

( $S_{3}$ denotes the group of permutations of a three-element set.) If we consider $b$ as a parameter in $\mathcal{N}_{(0,1, \infty)}$, then $g_{1}$ is the symmetry with respect to the unit circle composed with the symmetry with respect to the real line and $g_{3}$ is the symmetry with respect to the circle $\{z:|z-1|=1\}$ composed with the symmetry with respect to the real line. See Fig. 1a.

Now we describe the orbifold structure of $\mathcal{F} /\left\langle g_{1}, g_{2}\right\rangle$. Let

$$
H_{1}(a, b)=(x(a, b), y(b)) \text {, }
$$

where

$$
x(a, b)=a \frac{(2 b-1)^{2}}{b}+\frac{1}{a} \cdot \frac{(b-2)^{2}}{b}, \quad y(b)=\frac{(b-1)^{2}}{b}
$$


(a)

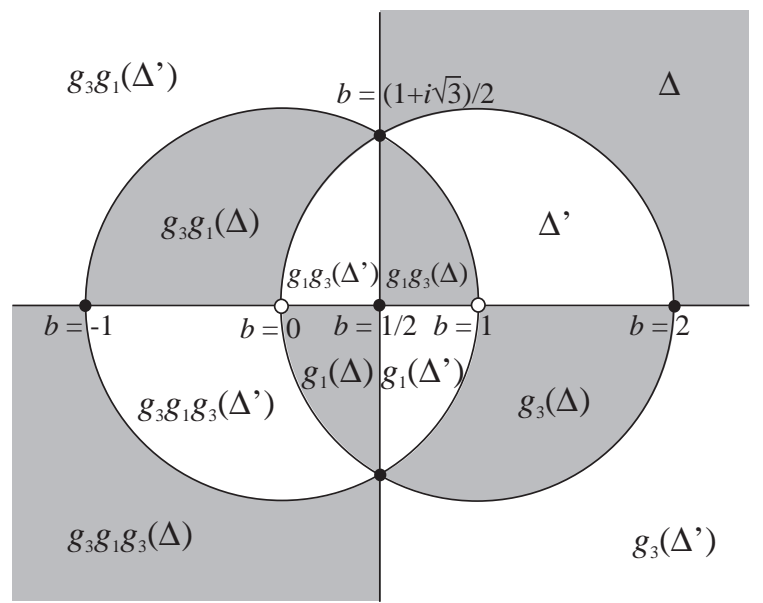

The action of $\left\langle g_{1}, g_{3}\right\rangle$ on the plane $\{(1, b): b \in \mathbb{C} \backslash\{0,1\}\}$

(b)

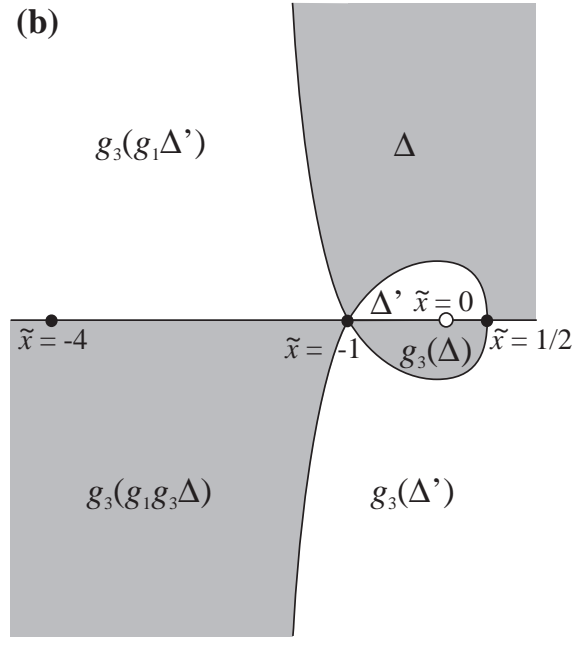

The action of $g_{3}$ on the plane $\{(\widetilde{x}, 0): \widetilde{x} \in \mathbb{C} \backslash\{0\}\}$ (the quotient space of the plane (a) under the action of $g_{1}$ )

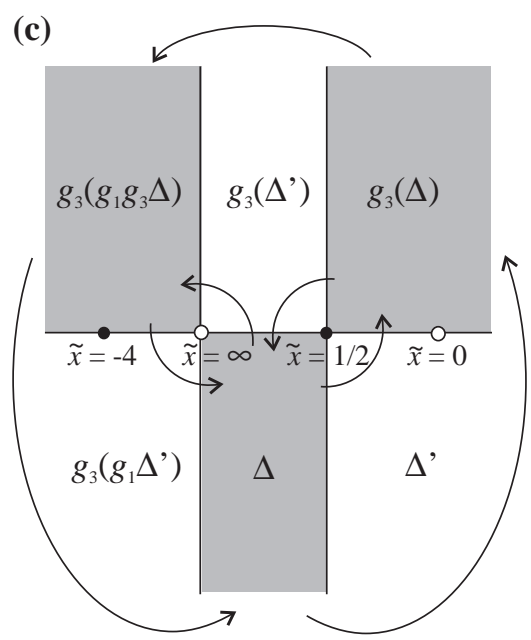

A schematic view of the plane (b) with $\widetilde{x}=-1$ moved to infinity

Fig. 1. The action of the Möbius group on Newton maps

for $a \in \mathbb{C} \backslash\{0\}, b \in \mathbb{C} \backslash\{0,1\}$. Using Lemma 3.2, it is easy to check that $H_{1}\left(a_{1}, b_{1}\right)=H_{1}\left(a_{2}, b_{2}\right)$ if and only if $\left(a_{1}, b_{1}\right)$ and $\left(a_{2}, b_{2}\right)$ are in the same orbit of the action of $\left\langle g_{1}, g_{2}\right\rangle$. Note that $H_{1}((\mathbb{C} \backslash\{0\}) \times(\mathbb{C} \backslash\{0,1\}))=X_{1}$, where

$$
X_{1}=(\mathbb{C} \times(\mathbb{C} \backslash\{0\})) \backslash\{(0,1 / 2)\} .
$$


(Here $\mathbb{C} \times\{0\}$ corresponds to $\left\{(a, b):(b-1)^{2} / b=0\right\}=\{(a, b): b=1\}$ and the point $(0,1 / 2)$ corresponds to $b=2, a \rightarrow 0$ and $b=1 / 2, a \rightarrow \infty$.) By general topology, $\mathcal{F} /\left\langle g_{1}, g_{2}\right\rangle$ is homeomorphic to $X_{1}$.

This together with Lemma 3.2 implies that $\mathcal{M}(\mathcal{F})$ is the quotient space of $X_{1}$ under the action of $g_{3}$ on maps with three supersinks (Newton maps). Note that Newton maps in $X_{1}$ correspond to the set

$$
\left\{\left(\frac{(2 b-1)^{2}+(b-2)^{2}}{b}, b+\frac{(b-1)^{2}}{b}\right)\right\}_{b \in \mathbb{C} \backslash\{0,1\}}=\{(5 y(b)+2, y(b))\}_{b \in \mathbb{C} \backslash\{0,1\}} .
$$

Apply an affine change of coordinates in $\mathbb{C} \times \mathbb{C}$ putting $\widetilde{x}=y, \widetilde{y}=x-$ $5 y-2$. In the coordinates $(\widetilde{x}, \widetilde{y})$ the Newton maps in $X_{1}$ form the punctured complex plane

$$
\{\widetilde{y}=0\}=\{(\widetilde{x}, 0): \widetilde{x} \in \mathbb{C} \backslash\{0\}\} .
$$

Recall that the action of $\left\langle g_{1}, g_{3}\right\rangle$ on $\mathcal{N}_{(0,1, \infty)}$ is isomorphic to the action of $S_{3}$ (see Fig. 1a). Using Lemma 3.2, it is easy to check that if we define

$$
G(b)=\frac{(2 b-1)^{2}(b-2)^{2}(b+1)^{2}}{b^{2}(b-1)^{2}}
$$

for $b \in \mathbb{C} \backslash\{0,1\}$, then $G\left(b_{1}\right)=G\left(b_{2}\right)$ if and only if $b_{1}$ and $b_{2}$ are in the same orbit of the action of $\left\langle g_{1}, g_{3}\right\rangle$ on $\mathcal{N}_{(0,1, \infty)}$. Moreover, $G(\mathbb{C} \backslash\{0,1\})=\mathbb{C}$. Now we can determine the orbifold structure of $\mathcal{M}(\mathcal{F}) \simeq \mathcal{F} /\left\langle g_{1}, g_{2}, g_{3}\right\rangle$. For $a \in \mathbb{C} \backslash\{0\}, b \in \mathbb{C} \backslash\{0,1\}$ define

$$
H(a, b)=(G(b), \widetilde{y}(a, b), y(b) \widetilde{y}(a, b)),
$$

where $\widetilde{y}(a, b)=x(a, b)-5 y(b)-2$ and $x(a, b), y(b)$ are defined in (4). Note that if $\widetilde{y}(a, b) \neq 0$, then $H\left(a_{1}, b_{1}\right)=H\left(a_{2}, b_{2}\right)$ if and only if $H_{1}\left(a_{1}, b_{1}\right)=$ $H_{1}\left(a_{2}, b_{2}\right)$. Moreover, $\widetilde{y}(a, b)=0$ means that $f_{a, b}$ is a Newton map and then $H\left(a_{1}, b_{1}\right)=H\left(a_{2}, b_{2}\right)$ if and only if $G\left(b_{1}\right)=G\left(b_{2}\right)$. By Lemma 3.2, this implies that $H\left(a_{1}, b_{1}\right)=H\left(a_{2}, b_{2}\right)$ if and only if $\left(a_{1}, b_{1}\right)$ and $\left(a_{2}, b_{2}\right)$ are in the same orbit of the action of $\left\langle g_{1}, g_{2}, g_{3}\right\rangle$.

Let

$$
X=H((\mathbb{C} \backslash\{0\}) \times(\mathbb{C} \backslash\{0,1\})) \cup\{v\},
$$

where $v=(0,-9 / 2,-9 / 4)$ corresponds to the point $(x, y)=(0,1 / 2)$ (see the definition of $X_{1}$ ). By general topology and Corollary 3.3, we obtain

Corollary 3.4. $\mathcal{M}(\mathcal{F}) \simeq \mathcal{F} /\left\langle g_{1}, g_{2}, g_{3}\right\rangle$ is homeomorphic to $X \backslash\{v\}$.

Using the definition of $H$, by an easy calculation one can find a polynomial $F: \mathbb{C}^{3} \rightarrow \mathbb{C}$ such that all points $\left(z_{1}, z_{2}, z_{3}\right) \in \mathbb{C}^{3}$ from $X$ satisfy the equation

$$
F\left(z_{1}, z_{2}, z_{3}\right)=0 .
$$


On the other hand, one can easily check that for any given point $\left(z_{1}, z_{2}, z_{3}\right) \in$ $\mathbb{C}^{3}$ such that (6) holds, either $\left(z_{1}, z_{2}, z_{3}\right)=v$ or we can find $a \in \mathbb{C} \backslash\{0\}$ and $b \in \mathbb{C} \backslash\{0,1\}$ such that $H(a, b)=\left(z_{1}, z_{2}, z_{3}\right)$. Therefore, we obtain

Corollary 3.5. The set $X$ is an algebraic set in $\mathbb{C}^{3}$ of complex dimension two.

Recall that the point $v$ corresponds to $(x, y)=(0,1 / 2)$ (in the coordinates $(\widetilde{x}, \widetilde{y})$ it is equal to $(1 / 2,-9 / 2))$. Hence $v$ represents the values $b=2, a \rightarrow 0$ and $b=1 / 2, a \rightarrow \infty$. Note that if in (1) we let $\alpha \rightarrow 0$, $\beta \rightarrow 0$, then $b \rightarrow 1 / 2, a \rightarrow \infty$. Hence $v$ corresponds to $\alpha=\beta=0$, which is the unique point of $\mathcal{M}(\mathfrak{F}) \backslash \mathcal{M}(\mathcal{F})$. Therefore, we can identify $\mathcal{M}(\mathfrak{F})$ with the set $X$. Note that the Newton maps in $X$ correspond to the set

$$
\{H(a, b): \widetilde{y}(a, b)=0\}=\{(G(b), 0,0): b \in \mathbb{C} \backslash\{0,1\}\}=\mathbb{C} \times\{0\} \times\{0\} .
$$

Take a closer look at the action of $g_{3}$ on Newton maps in $X_{1} \simeq \mathcal{F} /\left\langle g_{1}, g_{2}\right\rangle$ (it is presented in Fig. 1b,c). It is easy to compute that on the plane $\{(\widetilde{x}, 0)\}$, $g_{3}$ identifies the points $\widetilde{x}=(b-1)^{2} / b, \widetilde{x}=1 /(b(b-1))$ and $\widetilde{x}=b^{2} /(1-b)$. A generic orbit consists of three points. The exceptional points are $\widetilde{x}=1 / 2$, which is identified only with $\widetilde{x}=-4$ (here $b=-1,1 / 2,2$ and the map has a double supersink) and $\widetilde{x}=-1$, which is not identified with any other point (here $b=(1 \pm i \sqrt{3}) / 2) \cdot g_{3}$ acts like rotation through $2 \pi / 3$ near $\widetilde{x}=-1$ and like rotation through $\pi$ near $\widetilde{x}=1 / 2, \infty$ (see Fig. 1b,c).

For $z \in \mathbb{C}$ let

$$
X_{z}=\left\{\left(z_{1}, z_{2}, z_{3}\right) \in X: z_{1}=z\right\} .
$$

Note that $X_{z}=\{H(a, b): G(b)=z, a \in \mathbb{C} \backslash\{0\}\}$ (for $z=0$ we must add the point $v$ ). Hence $X_{z}$ corresponds to maps with fixed location of all critical points; moving the parameter $a$ we change the location of the critical values (see (3)).

Definition 3.6. Let $\mathbb{C}_{(k)}$ be the union of $k$ complex planes with exactly one common point. For instance, we can take

$$
\mathbb{C}_{(k)}=\left\{\left(z_{1}, z_{2}\right) \in \mathbb{C} \times \mathbb{C}: \prod_{j=1}^{k}\left(z_{1}-j z_{2}\right)=0\right\} .
$$

The above description of the action of $g_{3}$ on Newton maps implies that if $z \neq 0,-27$ (i.e. $b \neq-1,1 / 2,2,(1 \pm i \sqrt{3}) / 2$, which means $\widetilde{x} \neq-4,-1,1 / 2)$, then $X_{z}$ is homeomorphic to $\mathbb{C}_{(3)}$. In particular, this implies that $X$ is not a manifold. For $z=0$ (i.e. $b=-1,1 / 2,2, \widetilde{x}=-4,1 / 2$ ), the set $X_{0}$ is homeomorphic to $\mathbb{C}_{(2)}$. Moreover, $X_{0}$ corresponds to the maps with a double critical point, where one of the planes from $\mathbb{C}_{(2)}$ (for $\widetilde{x}=1 / 2$ ) represents the maps with a double supersink, hence conformally conjugate to polynomials. Finally, for $z=-27(b=(1 \pm i \sqrt{3}) / 2, \widetilde{x}=-1)$, the set $X_{-27}$ is 
homeomorphic to $\mathbb{C}$. It is easy to check that the point $(-27,0,0)$ corresponds to Newton's method for the polynomial $z^{3}-1$.

In this way we have proved

TheOREM 3.7. $\mathcal{M}(\mathfrak{F})$ can be identified with the set $X \subset \mathbb{C}^{3}$ defined in (5). The set $X$ is an algebraic set of complex dimension two. Moreover,

(a) $\mathbb{C} \times\{0\} \times\{0\}$ forms a set of singular points in $X$ and corresponds to the Newton maps in $\mathcal{M}(\mathfrak{F})$.

(b) For every $z \in \mathbb{C} \backslash\{0,-27\}, X_{z}$ is homeomorphic to $\mathbb{C}_{(3)}$.

(c) $X_{0}$ is homeomorphic to $\mathbb{C}_{(2)}$ and corresponds to the maps with a double critical point (one of the planes from $\mathbb{C}_{(2)}$ represents the polynomials).

(d) $X_{-27}$ is homeomorphic to $\mathbb{C}$ and $(-27,0,0)$ corresponds to Newton's method for the polynomial $z^{3}-1$.

REMARK. Note that apart from holomorphic conjugacy, $f_{a, b}$ is conjugate to $f_{\bar{a}, \bar{b}}$ by $z \mapsto \bar{z}$.

\section{The family $\mathcal{F}_{2}$}

4.1. Definitions and basic properties. In this section we study the family of maps $f_{a, b} \in \mathcal{F}$ for which the critical point 1 is periodic with period two. By (3), this condition is equivalent to

$$
b=-\frac{a^{2}+a-1}{a\left(a^{2}-a-1\right)} .
$$

This defines a one-parameter family $\mathcal{F}_{2} \subset \mathcal{F}$. Recall that $f_{a, b}$ is conjugate to $f_{1 / a, 1 / b}$ by $z \mapsto 1 / z$. To make this symmetry easier to notice (especially in figures), we replace $a$ by another parameter $\lambda$, so that for $f_{\lambda} \in \mathcal{F}_{2}, f_{\lambda}$ is conjugate to $f_{-\lambda}$. Let $\lambda=(a-1) /(a+1)$. Then

$$
a=\frac{1+\lambda}{1-\lambda}, \quad b=\frac{(1-\lambda)\left(\lambda^{2}-4 \lambda-1\right)}{(1+\lambda)\left(\lambda^{2}+4 \lambda-1\right)} .
$$

Let $f_{\lambda}=f_{a, b}$ for $a, b$ as in (7). Substituting (7) in (2) we obtain

$$
f_{\lambda}(z)=\frac{\lambda+1}{\lambda-1} z^{2} \frac{(\lambda-1)\left(\lambda^{2}-4 \lambda-1\right) z-\left(3 \lambda^{3}-5 \lambda^{2}+9 \lambda+1\right)}{\left(3 \lambda^{3}+5 \lambda^{2}+9 \lambda-1\right) z-(\lambda+1)\left(\lambda^{2}+4 \lambda-1\right)} .
$$

Since $f_{a, b}$ is conjugate to $f_{1 / a, 1 / b}$ by $z \mapsto 1 / z$, and $\lambda=(a-1) /(a+1), f_{\lambda}$ is conjugate to $f_{-\lambda}$ by $z \mapsto 1 / z$. These are all conformal conjugations between maps from $\mathcal{F}_{2}$ except the case when $u$ is periodic with period two (which holds for a finite number of parameters $\lambda$ ). Moreover, $f_{\lambda}$ is conjugate to $f_{\bar{\lambda}}$ by $z \mapsto \bar{z}$.

In Section 3 we described singular parameters $a, b$ in the family $\mathcal{F}$. Using this together with (7) one can characterize singular parameters $\lambda$. 
For $\lambda_{1}=-1, f_{\lambda}$ degenerates to the constant 0 , and analogously for $\lambda=-\lambda_{1}, f_{\lambda}$ is the constant $\infty$. Assume $\lambda \neq \pm \lambda_{1}$. The condition $b=\infty$ is equivalent to $\lambda^{2}+4 \lambda-1=0$, which gives $\lambda_{2}=-2-\sqrt{5}$ and $\lambda_{3}=-2+\sqrt{5}$. Recall that for these parameters, $f_{\lambda}$ is the quadratic polynomial $\frac{1+\lambda}{1-\lambda} z(2-z)$ and 0 is not a critical point. Similarly, $b=0$ is equivalent to $\lambda^{2}-4 \lambda-1=0$, i.e. $\lambda=-\lambda_{2},-\lambda_{3}$. Here $f_{\lambda}$ becomes the map $\frac{1+\lambda}{1-\lambda} \cdot \frac{z^{2}}{2 z-1}$ of degree two and $\infty$ is not a critical point. Finally, the condition $b=1$ means that $\lambda=\lambda_{4}, \pm \lambda_{5}$, where $\lambda_{4}=0, \lambda_{5}=\sqrt{3} i$ and $f_{\lambda}$ is the quadratic polynomial $\frac{1+\lambda}{1-\lambda} z^{2}$, where 1 is not a critical point. By (7), if $\lambda$ tends to $\pm \lambda_{2}, \ldots, \pm \lambda_{5}$, then $a$ tends to some finite non-zero number. Hence Lemma 2.2 gives

Corollary 4.1. • If $\lambda \rightarrow \widetilde{\lambda}$ for $\tilde{\lambda}=\lambda_{2}, \lambda_{3}$, then $f_{\lambda}(z) \rightarrow \frac{1+\widetilde{\lambda}}{1-\tilde{\lambda}} z(2-z)$ uniformly in the spherical metric on every set $U \subset \widehat{\mathbb{C}}$ such that $0 \notin \operatorname{cl} U$.

- If $\lambda \rightarrow \widetilde{\lambda}$ for $\widetilde{\lambda}=-\lambda_{2},-\lambda_{3}$, then $f_{\lambda}(z) \rightarrow \frac{1+\widetilde{\lambda}}{1-\tilde{\lambda}} \cdot \frac{z^{2}}{2 z-1}$ uniformly in the spherical metric on every set $U \subset \widehat{\mathbb{C}}$ such that $\infty \notin \operatorname{cl} U$.

- If $\lambda \rightarrow \tilde{\lambda}$ for $\tilde{\lambda}=\lambda_{4}, \pm \lambda_{5}$, then $f_{a, b}(z) \rightarrow \frac{1+\tilde{\lambda}}{1-\tilde{\lambda}} z^{2}$ uniformly in the spherical metric on every set $U \subset \widehat{\mathbb{C}}$ such that $1 \notin \mathrm{cl} U$.

Note also that $\lambda=\infty$ is not a singular parameter and $f_{\infty}(z)=z^{2} \frac{z-3}{3 z-1}$.

From the above considerations we conclude that the family

$$
\mathcal{F}_{2}=\left\{f_{\lambda}: \lambda \in \Lambda\right\}, \quad \Lambda=\widehat{\mathbb{C}} \backslash\left\{ \pm \lambda_{1}, \ldots, \pm \lambda_{5}\right\},
$$

is the family of all rational maps of degree three with superattracting fixed points $0, \infty$ and critical point 1 of period two.

We study the behaviour of the critical point $u$ of $f_{\lambda}$ with respect to the parameter $\lambda \in \Lambda$. We write $u=u_{\lambda}$. By (3) and (7),

$$
\begin{gathered}
u_{\lambda}=\frac{(\lambda+1)\left(\lambda^{2}+4 \lambda-1\right)\left(3 \lambda^{3}-5 \lambda^{2}+9 \lambda+1\right)}{(\lambda-1)\left(\lambda^{2}-4 \lambda-1\right)\left(3 \lambda^{3}+5 \lambda^{2}+9 \lambda-1\right)} \\
f_{\lambda}\left(u_{\lambda}\right)=-\frac{(\lambda+1)^{2}\left(\lambda^{2}+4 \lambda-1\right)\left(3 \lambda^{3}-5 \lambda^{2}+9 \lambda+1\right)^{3}}{(\lambda-1)^{2}\left(\lambda^{2}-4 \lambda-1\right)\left(3 \lambda^{3}+5 \lambda^{2}+9 \lambda-1\right)^{3}} .
\end{gathered}
$$

Definition 4.2. For $\zeta=0, \infty, 1, f_{\lambda}(1)$ let $B(\zeta)$ be the immediate basin of attraction to $\zeta$ and let

$$
\Lambda_{\zeta}=\left\{\lambda \in \Lambda: u_{\lambda} \in B(\zeta)\right\} .
$$

Recall that $f_{\bar{\lambda}}$ is conjugate to $f_{\lambda}$ by $z \mapsto \bar{z}$ and $f_{-\lambda}$ is conjugate to $f_{\lambda}$ by $z \mapsto 1 / z$. This means that symmetry with respect to the real axis in the parameter plane does not change the behaviour of $u_{\lambda}$. Similarly, symmetry with respect to the origin of the parameter plane only changes the role of 0 


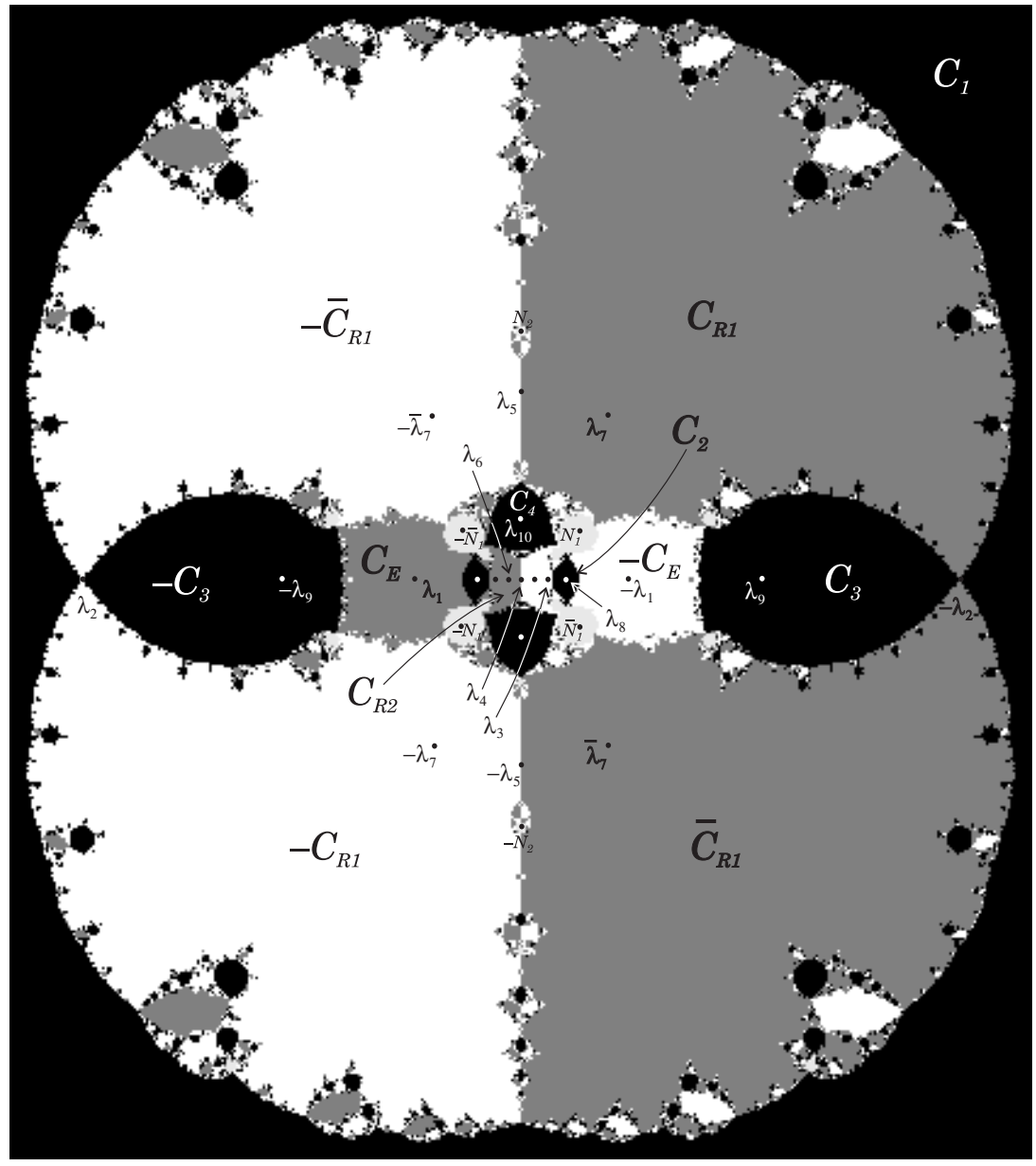

white: $u_{\lambda}$ is in the basin of $\infty \quad$ black: $u_{\lambda}$ is in the basin of 1 gray: $u_{\lambda}$ is in the basin of 0 light gray: else $\pm N_{1}, \pm \bar{N}_{1}, \pm N_{2}$ : Newton maps

$$
\begin{array}{lllll}
\lambda_{1}=-1 & \lambda_{2}=-2-\sqrt{5} & \lambda_{3}=-2+\sqrt{5} & \lambda_{4}=0 & \lambda_{5}=\sqrt{3} i \\
\lambda_{6} \approx-0.10 & \lambda_{7} \approx 0.89+1.55 i & \lambda_{8}=\sqrt{5} / 5 & \lambda_{9}=\sqrt{5} & \lambda_{10}=(\sqrt{3} / 3) i
\end{array}
$$

Fig. 2. Parameter plane for $\mathcal{F}_{2}$

and $\infty$. In particular, this implies

$$
\begin{gathered}
\Lambda_{0}=\bar{\Lambda}_{0}, \quad \Lambda_{\infty}=\bar{\Lambda}_{\infty}, \quad \Lambda_{0}=-\Lambda_{\infty}, \\
-\Lambda_{1}=\Lambda_{1}=\bar{\Lambda}_{1}, \quad-\Lambda_{f_{\lambda}(1)}=\Lambda_{f_{\lambda}(1)}=\bar{\Lambda}_{f_{\lambda}(1)},
\end{gathered}
$$

where $-A=\{-z: z \in A\}$ and $\bar{A}=\{\bar{z}: z \in A\}$ (see Fig. 2). 
We are going to describe all components of $\Lambda_{0}, \Lambda_{\infty}, \Lambda_{1}$ and $\Lambda_{f_{\lambda}(1)}$. We will show that there are a finite number of them, and each has a "centre point", which can be either a singular parameter or a point from $\Lambda$. We will also determine which component is "exotic" and which is "regular". To explain what we mean, we need to state some definitions.

In [P1] Feliks Przytycki introduced the following notion of exotic basins:

Definition 4.3. Let $f$ be a rational map of degree $d \geq 3$. Suppose that $f$ has an attracting fixed point $s$ and let $B(s)$ be the immediate basin of attraction of $s$. Then we call $B(s)$ an exotic basin if the following hold:

(i) $B(s)$ is completely invariant, i.e. $f^{-1}(B(s))=B(s)$,

(ii) $B(s)$ is non-simply connected,

(iii) $B(s)$ contains less than $d$ critical points counted with multiplicity.

REMARK. An equivalent formulation of the condition (i) is to say that the entire basin of $s$ is connected or that $f$ has degree $d$ on $B(s)$.

Note that if $B(s)$ is simply connected, then it contains exactly $\left.\operatorname{deg} f\right|_{B(s)}$ - 1 critical points counted with multiplicity because then $\left.f\right|_{B(s)}$ is conjugate to a Blaschke product on the unit disc. In [P1] it is proved that if $f$ is expanding on the Julia set and $B(s)$ contains all but one critical points or values counted without multiplicity, then $f$ has degree $d$ on $B(s)$, and moreover, the component of the set of rational maps of degree $d$ expanding on the Julia set which contains $f$, also contains a polynomial. On the other hand, in [P1] examples are given of basins containing $k$ critical points counted with multiplicity for an arbitrary $k=2, \ldots, 2 d-2$. Note that the basin of $\infty$ for a polynomial of degree $d$ cannot be exotic. This follows from the observation that $\infty$ is the critical point of degree $d-1$ and a well-known fact that if $B(s)$ is non-simply connected, then there are at least two critical points in it (counted without multiplicity).

The following fact was proved in [Ba]:

THEOREM 4.4. Let $f$ be a rational map of degree $d \geq 3$ and let $s$ be an attracting fixed point. Suppose that the immediate basin of attraction $B(s)$ contains at least $2 d-4$ critical points or values counted with multiplicity. Then $f$ has degree $d$ on $B(s)$. Moreover, for arbitrary $d \geq 3$ and $k=$ $1, \ldots, 2 d-5$ there exists a rational map $f$ with an attracting fixed point $s$ such that $B(s)$ contains exactly $k$ critical points counted with multiplicity and the degree of $f$ on $B(s)$ is strictly less than $d$.

Let us now return to our situation and consider a map $f_{\lambda} \in \mathcal{F}_{2}$. Only one critical point $u_{\lambda}$ is "free" and can lie in the immediate basin of attraction of a sink. If $u_{\lambda} \in B(\xi)$ for an attracting fixed point $\xi \neq 0, \infty$, then $B(\xi)$ contains only one critical point, so it is simply connected and $\left.\operatorname{deg} f_{\lambda}\right|_{B(\xi)}=2$. 


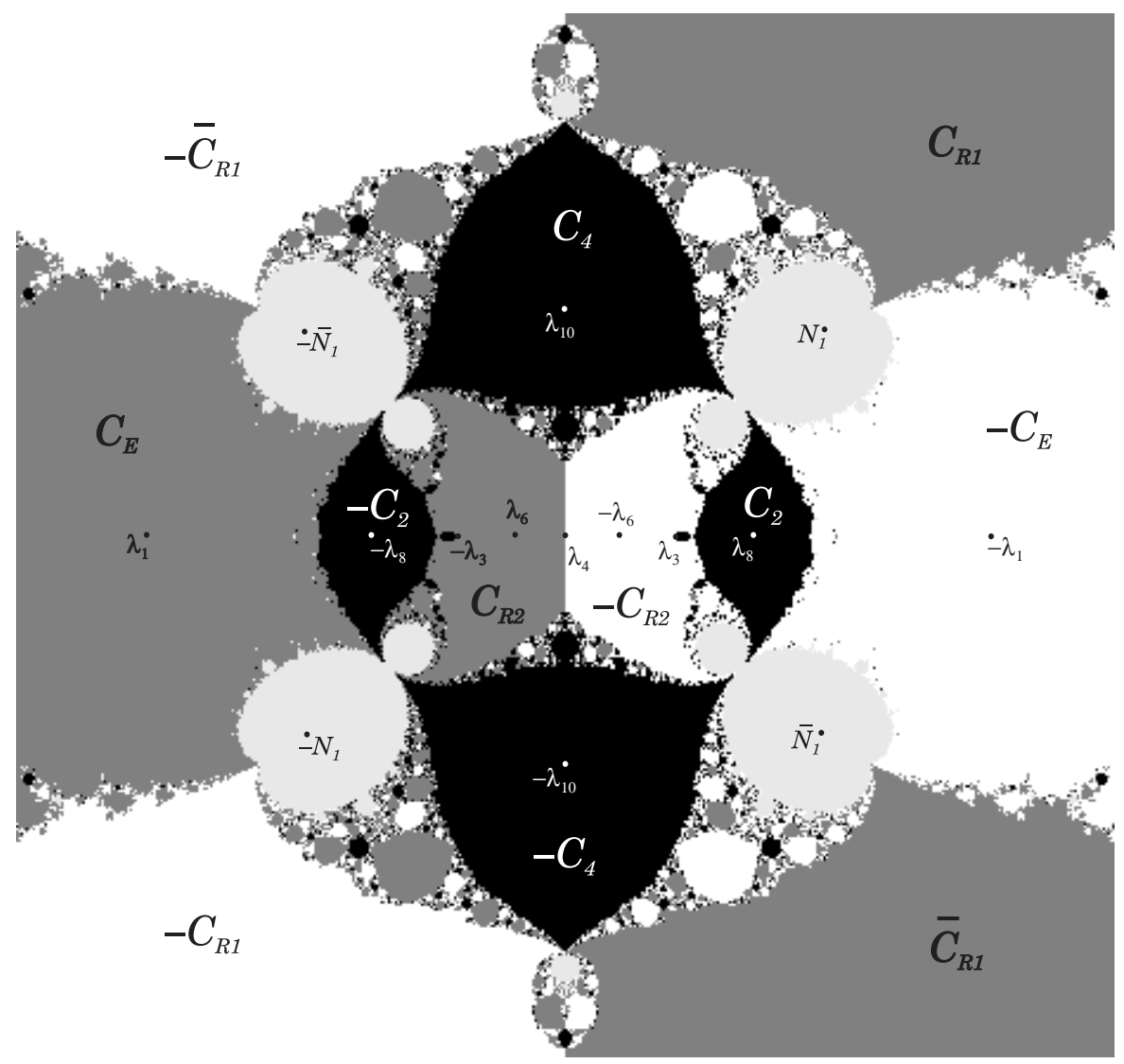

Fig. 3. A magnification of the parameter plane around 0 . The four light-gray sets similar to the Mandelbrot set are the regions where $u_{\lambda}$ is in the basin of an attracting periodic orbit disjoint from $0,1, \infty$.

Topologically this is the situation of a Newton map (i.e. $f_{\lambda}\left(u_{\lambda}\right)=u_{\lambda}$ ) (see Subsection 4.4).

For $B(0)$ and $B(\infty)$, Theorem 4.4 implies the following:

Corollary 4.5. Let $\zeta=0, \infty$.

- If $u_{\lambda} \notin B(\zeta)$, then $B(\zeta)$ is simply connected and $f_{\lambda}$ has degree two on $B(\zeta)$

- If $u_{\lambda} \in B(\zeta)$, then $f_{\lambda}$ has degree three on $B(\zeta)$. In this case $B(\zeta)$ is exotic if and only if $B(\zeta)$ is non-simply connected.

Moreover, $u_{\lambda} \in B(\zeta)$ if and only if $f_{\lambda}\left(u_{\lambda}\right) \in B(\zeta)$.

Let $C$ be a component of $\Lambda_{\zeta}$ for $\zeta=0, \infty$. Then $f_{\lambda}$ is hyperbolic and structurally stable for small perturbations (see [MSS]). Therefore either $B(\zeta)$ 
is exotic for all $\lambda \in C$, or it is non-exotic for all $\lambda \in C$. Hence we can state the following definition:

Definition 4.6. If $B(\zeta)$ is exotic for $\lambda \in C$, then we call $C$ an exotic component. Otherwise we call it a regular component.

By Corollary 4.5, $C$ is exotic if and only if $B(\zeta)$ is non-simply connected for $\lambda \in C$. Moreover, the following holds:

LEMma 4.7. Let $C$ be a component of $\Lambda_{\zeta}$ for $\zeta=0, \infty$. If there exists $\lambda \in C$ such that $\zeta$ is a double critical point for $f_{\lambda}\left(\right.$ i.e. $u_{\lambda}=\zeta$ ), then $C$ is regular.

Proof. For the map $f_{\lambda}, \zeta$ is the unique critical point in $B(\zeta)$. Hence $B(\zeta)$ is simply connected.

This fact will imply that every exotic component has a singular parameter as an isolated point in its boundary (see Theorem 4.15).

Using (3) and (7), it is easy to check that the following holds:

Proposition 4.8. If $\lambda \in \Lambda$, then:

- $u_{\lambda}=0 \Leftrightarrow b=1 / 2 \Leftrightarrow 3 \lambda^{3}-5 \lambda^{2}+9 \lambda+1=0$. This holds when $\lambda=\lambda_{6}, \lambda_{7}, \bar{\lambda}_{7}$, where $\lambda_{6} \approx-0.1046, \lambda_{7} \approx 0.8856+1.5495 i$. Moreover, $\lambda_{6}, \lambda_{7}, \bar{\lambda}_{7}$ lie in regular components of $\Lambda_{0}$.

- $u_{\lambda}=\infty \Leftrightarrow b=2 \Leftrightarrow \lambda=-\lambda_{6},-\lambda_{7},-\bar{\lambda}_{7}$. Moreover, $-\lambda_{6},-\lambda_{7},-\bar{\lambda}_{7}$ lie in regular components of $\Lambda_{\infty}$.

- $u_{\lambda}=1 \Leftrightarrow b=-1 \Leftrightarrow \lambda=\infty, 5 \lambda^{2}-1=0$. This holds when $\lambda=\infty, \pm \lambda_{8}$, where $\lambda_{8}=\sqrt{5} / 5$.

- $u_{\lambda}=f_{\lambda}(1) \Leftrightarrow\left(\lambda^{2}-5\right)\left(3 \lambda^{2}+1\right)=0$. This holds when $\lambda= \pm \lambda_{9}, \pm \lambda_{10}$, where $\lambda_{9}=\sqrt{5}, \lambda_{10}=(\sqrt{3} / 3) i$.

We are going to prove the following theorem, which classifies all components of $\Lambda_{\zeta}$ :

THEOREM 4.9. There are exactly four components of $\Lambda_{0}$ : three regular, $C_{R 1}, \bar{C}_{R 1}, C_{R 2}$, and one exotic, $C_{E}$. Analogously, $\Lambda_{\infty}$ consists of three regular components, $-C_{R 1},-\bar{C}_{R 1},-C_{R 2}$, and one exotic, $-C_{E}$. There are three components of $\Lambda_{1}: C_{1}, C_{2}$, and $-C_{2}$, and four components of $\Lambda_{f_{\lambda}(1)}$ : $C_{3},-C_{3}, C_{4}$ and $-C_{4}$. All regular components are topological discs and all exotic components are punctured discs. Moreover,

- $\lambda_{1}$ is an isolated point in $\partial C_{E}$,

- $\lambda_{2} \in \partial\left(-C_{R 1}\right) \cap \partial\left(-\bar{C}_{R 1}\right) \cap \partial C_{1} \cap \partial\left(-C_{3}\right) \cap\left(\widehat{\mathbb{C}} \backslash \operatorname{cl} \Lambda_{0}\right)$,

- $\lambda_{3} \in \partial\left(-C_{R 2}\right) \cap\left(\widehat{\mathbb{C}} \backslash \operatorname{cl} \Lambda_{0}\right)$,

- $\lambda_{4} \in \partial C_{R 2} \cap \partial\left(-C_{R 2}\right)$,

- $\lambda_{5} \in \partial C_{R 1} \cap \partial\left(-\bar{C}_{R 1}\right)$,

- $\lambda_{6} \in C_{R 2}, \lambda_{7} \in C_{R 1}, \lambda_{8} \in C_{2}, \lambda_{9} \in C_{3}, \lambda_{10} \in C_{4}, \infty \in C_{1}$. 
By symmetry, $-\lambda_{1}, \ldots,-\lambda_{10}, \bar{\lambda}_{7},-\bar{\lambda}_{7}$ have analogous properties. See Figs. 2 and 3 .

First we describe the behaviour of $u_{\lambda}$ for $\lambda$ in the neighbourhoods of the singular parameters $\pm \lambda_{1}, \ldots, \pm \lambda_{5}$.

\subsection{Singular parameters}

Proposition 4.10. $\lambda_{1}$ (resp. $\left.-\lambda_{1}\right)$ is an isolated point of $\partial C_{E}$ (resp. $\partial\left(-C_{E}\right)$ ), where $C_{E}$ is an exotic component of $\Lambda_{0}$ and $-C_{E}$ is an exotic component of $\Lambda_{\infty}$.

Proof. Consider parameters $\lambda$ in a small neighbourhood of $\lambda_{1}=-1$. By (9), we have $\left|f_{\lambda}\left(u_{\lambda}\right)\right|<c\left|\lambda-\lambda_{1}\right|^{2}$ for some constant $c$ independent of $\lambda$. On the other hand, (8) implies that if $|z|<c\left|\lambda-\lambda_{1}\right|^{2}$, then $\left|f_{\lambda}(z)\right|<c_{1}|z|^{2}$ for $c_{1}$ independent of $\lambda$. Hence for $\lambda$ close to $\lambda_{1}, f_{\lambda}\left(u_{\lambda}\right)$ is in $B(0)$. By Corollary 4.5, $u_{\lambda} \in B(0)$. Hence $\lambda_{1}$ is an isolated point of $\partial C$ for some component $C$ of $\Lambda_{0}$.

To see that $C$ is exotic consider real parameters $\lambda$ slightly less than $\lambda_{1}$. Then $f_{\lambda}(1)=(\lambda+1) /(\lambda-1)$ is a small positive real number. Let $x=f_{\lambda}^{-1}(0) \backslash\{0\}$. By (2), $x=2-1 / b$, so by (7), $x$ is a real number close to 2 . Hence $f_{\lambda}(1)$ lies between 0 and $x$. Moreover, $0, x \in B(0), f_{\lambda}(1), \infty \notin B(0)$ and $B(0)$ is symmetric with respect to the real axis. This implies that $B(0)$ is not simply connected, so $C$ is exotic (we denote it by $C_{E}$ ).

The second part follows from (10).
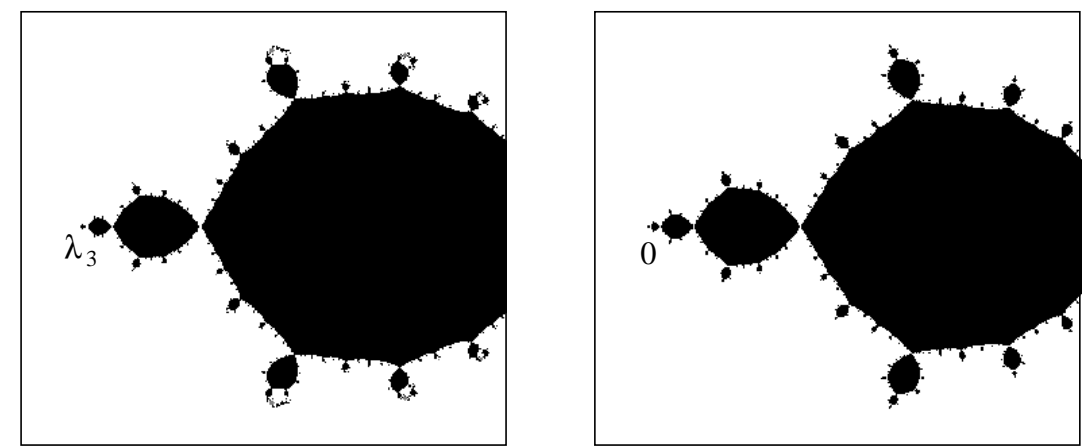

Fig. 4. A fragment of the $\lambda$-parameter plane near $\lambda_{3}$ (left) and a fragment of the filled-in Julia set of $\frac{1+\sqrt{5}}{2} z(2-z)$ near 0 (right)

Recall that near the singular values $\pm \lambda_{2}, \ldots, \pm \lambda_{5}$ the map $f_{\lambda}$ tends to some map of degree two with a supersink (hence conformally conjugate to a polynomial). Moreover, Corollary 4.1 implies that the convergence is uniform outside small neighbourhoods of resp. 0,1 or $\infty$. This is the reason the $\lambda$-parameter plane near the singular values $\pm \lambda_{2}, \ldots, \pm \lambda_{5}$ looks like the 
dynamical plane in the neighbourhoods of some points in the Julia sets of appropriate polynomials of degree two. In particular, $\lambda_{2}$ corresponds to 0 for the polynomial $\frac{1-\sqrt{5}}{2} z(2-z)$ and $\lambda_{3}$ corresponds to 0 for $\frac{1+\sqrt{5}}{2} z(2-z)$ (see Fig. 4). Both these polynomials are conformally conjugate to $z^{2}-1$. Furthermore, $\lambda_{4}$ corresponds to 1 for $z^{2}$, and $\lambda_{5}$ corresponds to 1 for $\frac{-1+\sqrt{3} i}{2} z^{2}$ (the latter polynomial is conjugate to $z^{2}$ ). By this similarity, we are able to describe the plane $\Lambda$ near the singular parameters.

Proposition 4.11. $\lambda_{2} \in \partial\left(-C_{R 1}\right) \cap \partial\left(-\bar{C}_{R 1}\right) \cap \partial C_{1} \cap \partial\left(-C_{3}\right) \cap\left(\widehat{\mathbb{C}} \backslash \operatorname{cl} \Lambda_{0}\right)$, where $-C_{R 1},-\bar{C}_{R 1}$ are regular components of $\Lambda_{\infty}, C_{1}$ is a component of $\Lambda_{1}$ and $-C_{3}$ is a component of $\Lambda_{f_{\lambda}(1)}$. Moreover, there exist $r_{0}>0$ and $0<\alpha<\pi / 2$ such that for $\lambda=\lambda_{2}+r e^{i \theta}$ and $0<r<r_{0}$ :

- if $\pi / 2-\alpha<\theta<\pi / 2+\alpha$, then $\lambda \in-\bar{C}_{R 1}$,

- if $-\pi / 2-\alpha<\theta<-\pi / 2+\alpha$, then $\lambda \in-C_{R 1}$,

- if $-\alpha<\theta<\alpha$, then $\lambda \in-C_{3}$,

- if $\pi-\alpha<\theta<\pi+\alpha$, then $\lambda \in C_{1}$.

By symmetry, $-\lambda_{2}$ has analogous properties.

Pr o of. Since (7) implies

$$
\frac{1}{b}=\frac{-15+7 \sqrt{5}}{8}\left(\lambda-\lambda_{2}\right)+\mathcal{O}\left(\left(\lambda-\lambda_{2}\right)^{2}\right)
$$

as $\lambda \rightarrow \lambda_{2}$, we will make estimates replacing $\lambda-\lambda_{2}$ for $\lambda \rightarrow \lambda_{2}$ by $1 / b$ for $b \rightarrow \infty$ (this will simplify the calculations). Using (2) and (7), one can compute that for any fixed $x \in \mathbb{C}$,

(11) if $z=x / b+\mathcal{O}\left(1 / b^{2}\right), \quad$ then $\quad f_{\lambda}(z)=\frac{(1-\sqrt{5}) x^{2}}{x+1} \cdot \frac{1}{b}+\mathcal{O}\left(1 / b^{2}\right)$.

Since for $|x|>2+\sqrt{5}+\varepsilon(\varepsilon>0)$ we have

$$
\left|\frac{(1-\sqrt{5}) x^{2}}{x+1}\right|>q|x|
$$

where $q>1$, this implies that for $\lambda$ sufficiently close to $\lambda_{2}$,

(12) if $z=x / b+\mathcal{O}\left(1 / b^{2}\right)$ for $|x|>2+\sqrt{5}+\varepsilon$, then $\left|f_{\lambda}(z)\right|>q|z|$,

where $q>1$ is a constant independent of $x, z, b$. By (3) and (7), for $\lambda$ close to $\lambda_{2}$,

$$
f_{\lambda}\left(u_{\lambda}\right)=4(\sqrt{5}-1) / b+\mathcal{O}\left(1 / b^{2}\right) .
$$

Since $4(\sqrt{5}-1)>2+\sqrt{5}$, the assumption of (12) is satisfied for $z=f_{\lambda}\left(u_{\lambda}\right)$. Moreover, (11) implies that $f_{\lambda}^{3}\left(u_{\lambda}\right)=A / b+\mathcal{O}\left(1 / b^{2}\right)$ for $A$ real and greater than $4(\sqrt{5}-1)$. Hence if we connect $f_{\lambda}\left(u_{\lambda}\right)$ with $f_{\lambda}^{3}\left(u_{\lambda}\right)$ by a straight line segment $I$, then the assumption of $(12)$ is satisfied for every point in $I$. 
Proceeding by induction, we show that for every large $M>0$ and small $\delta>0$ there exists $n_{0}$ such that for $z \in I$,

$$
f_{\lambda}^{n_{0}}(z)=C(z) / b+\mathcal{O}\left(1 / b^{2}\right),
$$

where $|C(z)|>M$ and $|\operatorname{Im}(C(z))|<\delta$. By the compactness of $I$, there exists a neighbourhood $U$ of $\lambda_{2}$ such that for every $\lambda \in U \cap \Lambda$ and for every $z \in I$,

$$
\left|f_{\lambda}^{n_{0}}(z)\right|>M /|b| \text { and }\left|\operatorname{Arg}\left(f_{\lambda}^{n_{0}}(z)\right)-\operatorname{Arg}(1 / b)\right|<\delta .
$$

Let $g(z)=\frac{1+\lambda_{2}}{1-\lambda_{2}} z(2-z)=\frac{1-\sqrt{5}}{2} z(2-z)$. Recall that $f_{\lambda} \rightarrow g$ as $\lambda \rightarrow \lambda_{2}$ and $g$ is conformally conjugate to $z^{2}-1$.

We now deal with the case $\lambda \in \Lambda_{\infty}$ (the cases $\lambda \in \Lambda_{1}$ and $\lambda \in \Lambda_{f_{\lambda}(1)}$ can be proved analogously). Denote by $B_{g}(\infty)$ the basin of attraction to $\infty$ for $g$. Note that 0 is the landing point of external rays in $B_{g}(\infty)$ with arguments $1 / 3$ and $2 / 3$. Let

$$
S_{\alpha}=\{z \in \mathbb{C} \backslash\{0\}: \pi / 2-\alpha<|\operatorname{Arg}(z)|<\pi / 2+\alpha\} .
$$

It is known that there exists $0<\alpha<\pi / 2$ such that $S_{\alpha} \subset B_{g}(\infty)$ (this follows e.g. from the fact that 0 is a repelling fixed point for $g$ ). Assume that this $\alpha$ is maximal. We show that for each $0<\beta<\alpha$ there exists a neighbourhood $U$ of $\lambda_{2}$ such that $u_{\lambda} \in B_{f_{\lambda}}(\infty)$ for $\lambda \in U \cap S_{\beta}$. Note that by Corollary 4.5, it suffices to show $f_{\lambda}\left(u_{\lambda}\right) \in B_{f_{\lambda}}(\infty)$. Fix $0<\beta<\alpha$. Take a small number $a>0$. Then for every $z$, if $z \in S_{\beta}$ and $|z|>a$, then $\operatorname{dist}(z, J(g))>d(a)$. Let $\Phi_{\infty}: B_{g}(\infty) \rightarrow \mathbb{D}$ be the Böttcher coordinates on $B_{g}(\infty)$ and for $0<r<1$ let $P_{r}$ be equipotential curves in $B_{g}(\infty)$, i.e. $P_{r}=\Phi_{\infty}^{-1}(\{z:|z|=r\})$. Recall that $g\left(P_{r}\right)=P_{r^{2}}$. Since $\operatorname{dist}\left(P_{r}, J(g)\right) \rightarrow 0$ as $r \rightarrow 1$, there exists $r_{0}<1$ such that $\{z: \operatorname{dist}(z, J(g))>d(a)\} \subset \bigcup_{r \leq r_{0}} P_{r}$. Let

$$
d_{0}=\inf _{r \leq r_{0}} \operatorname{dist}\left(P_{r}, P_{r^{2}}\right) \text {. }
$$

By definition, $d_{0}>0$. Now we prove that for $\lambda$ sufficiently close to $\lambda_{2}$,

$$
\text { if } z \in S_{\beta} \text { and }|z|>a \text {, then } z \in B_{f_{\lambda}}(\infty) \text {. }
$$

The idea is that $z$ is in $B_{g}(\infty)$ and $f_{\lambda}$ is so close to $g$ that the "error" will be too small to compensate for the attraction to $\infty$. Define $\operatorname{Err}_{\lambda}(z)=$ $f_{\lambda}(z)-g(z)$. Then by (2) and (7),

$$
\left|\operatorname{Err}_{\lambda}(z)\right|<c\left|\frac{z}{(2-b) z-1}\right|
$$

for some constant $c$ independent of $\lambda$ and $z$ provided $|z|<R$ for some large fixed $R$ (this assumption does not cause any problems because if $|z|$ is large, then obviously $\left.z \in B_{f_{\lambda}}(\infty)\right)$. Therefore there exist constants $c_{1}, c_{2}$, independent of $\lambda$ close to $\lambda_{2}$, such that

$$
\text { if } c_{1} /|b|<|z|<R, \text { then }\left|\operatorname{Err}_{\lambda}(z)\right|<c_{2} /|b| .
$$


Take $\lambda$ so close to $\lambda_{2}$ that $c_{1} /|b|<a$ and $c_{2} /|b|<d_{0}$. Then (16) and the definition of $d_{0}$ give

$$
\operatorname{cl} f_{\lambda}\left(\bigcup_{r \leq r_{0}} P_{r}\right) \subset \bigcup_{r \leq r_{0}} P_{r} .
$$

Therefore $\bigcup_{r \leq r_{0}} P_{r}$ is contained in $B_{f_{\lambda}}(\infty)$. In this way we have proved (15).

Now we show that for any fixed $0<\beta^{\prime}<\beta$, there exists a small $a>0$ and a large $M$ such that for every $\lambda$ sufficiently close to $\lambda_{2}$,

(17) if $z \in S_{\beta^{\prime}}$ and $M /|b|<|z|<a$, then $f_{\lambda}^{n_{1}}(z) \in S_{\beta}$ and $\left|f_{\lambda}^{n_{1}}(z)\right|>a$ for some $n_{1}$.

Since 0 is a repelling fixed point for $g$, there exist $a>0$ and $q>1$ such that for every $z$, if $|z|<a$, then

$$
|g(z)|>q|z| .
$$

Moreover, if $|z|<a$, then

$$
|| \operatorname{Arg}(g(z))|-| \operatorname{Arg}(z)||=|\operatorname{Arg}(2-z)| \leq c_{3}|z| .
$$

Take a large fixed number $M$. By (16), (18) and trigonometry, if $M /|b|<$ $|z|<a$, then

$$
|| \operatorname{Arg}\left(f_{\lambda}(z)\right)|-| \operatorname{Arg}(g(z))|| \leq \frac{c_{4}}{|b| \cdot|z|} .
$$

Take an arbitrary $z$ such that $M /|b|<|z|<a$. By (16) and (18), there exists a minimal $n_{1}$ such that $\left|f_{\lambda}^{n_{1}}\right|>a$. Moreover, if $M$ is sufficiently large, then for every $k=0, \ldots, n_{1}-1$,

$$
q^{k} \frac{M}{|b|}<\left|f_{\lambda}^{k}(z)\right|<\frac{a}{q^{n_{1}-1-k}} .
$$

Therefore, using (19) and (20), we obtain

$$
\begin{aligned}
|| \operatorname{Arg}\left(f_{\lambda}^{n_{1}}(z)\right)|-| \operatorname{Arg}(z)|| & \leq \sum_{k=0}^{n_{1}-1}|| \operatorname{Arg}\left(f_{\lambda}^{k+1}(z)\right)|-| \operatorname{Arg}\left(f_{\lambda}^{k}(z)\right) \| \\
& \leq c_{3} \sum_{k=0}^{n_{1}-1}\left|f_{\lambda}^{k+1}(z)\right|+\frac{c_{4}}{|b|} \sum_{k=0}^{n_{1}-1} \frac{1}{\left|f_{\lambda}^{k+1}(z)\right|}=\mathrm{I}+\mathrm{II} .
\end{aligned}
$$

By $(21)$,

$$
\begin{aligned}
& \mathrm{I}<c_{3} a \sum_{k=0}^{n_{1}-1} \frac{1}{q^{n_{1}-1-k}}<c_{3} a \frac{q}{q-1}, \\
& \mathrm{II}<\frac{c_{4}}{M} \sum_{k=0}^{n_{1}-1} \frac{1}{q^{k}}<\frac{c_{4}}{M} \cdot \frac{q}{q-1} .
\end{aligned}
$$


Hence for sufficiently small $a$ and large $M,|| \operatorname{Arg}\left(f_{\lambda}^{n_{1}}(z)\right)|-| \operatorname{Arg}(z)||$ is less than $\beta-\beta^{\prime}$, so $f_{\lambda}^{n_{1}}(z) \in S_{\beta}$. In this way we have proved (17).

By (14), if $\lambda$ is close to $\lambda_{2}$ and $\lambda \in S_{\beta^{\prime \prime}}$ for $\beta^{\prime \prime}$ slightly less than $\beta^{\prime}$, then the assumption of (17) is satisfied for all points from $f^{n_{0}}(I)$. Hence (17) and (15) give $f^{n_{0}+n_{1}}(I) \subset B_{f_{\lambda}}(\infty)$, so $f_{\lambda}\left(u_{\lambda}\right)$ can be connected to points from $B_{f_{\lambda}}(\infty)$ by a curve contained in the basin of $\infty$. This gives $f_{\lambda}\left(u_{\lambda}\right) \in B_{f_{\lambda}}(\infty)$ so $\lambda \in \Lambda_{\infty}$.

It remains to show that $\lambda$ is in a regular component of $\Lambda_{\infty}$, i.e. $B_{f_{\lambda}}(\infty)$ is simply connected. This will be done in Corollary 4.16.

We have proved the case $\lambda \in \Lambda_{\infty}$. As one can easily see, the proofs of the cases $\lambda \in \Lambda_{1}$ and $\lambda \in \Lambda_{f_{\lambda}(1)}$ can be done in a similar way. We omit the details.

It remains to prove that $\lambda_{2} \in \widehat{\mathbb{C}} \backslash \operatorname{cl} \Lambda_{0}$. This means that for $\lambda$ near $\lambda_{2}$, $u_{\lambda}$ cannot be in $B_{f_{\lambda}}(0)$. To prove this, suppose that $u_{\lambda} \in B_{f_{\lambda}}(0)$. Connect $f_{\lambda}\left(u_{\lambda}\right)$ to $f_{\lambda}^{2}\left(u_{\lambda}\right)$ by a simple curve $\gamma_{0} \subset B_{f_{\lambda}}(0)$ parameterized by $t \in[0,1]$ and define a curve $\gamma:[0, \infty) \rightarrow B_{f_{\lambda}}(0)$ setting $\gamma(t)=f_{\lambda}^{[t]}(\gamma(t-[t]))$. By definition, $\gamma$ connects $f_{\lambda}\left(u_{\lambda}\right)$ and 0 . Fix a small $\varepsilon>0$ and let

$$
\mathcal{T}=\{t \geq 0:|\gamma(t)|=(2+\sqrt{5}+\varepsilon) /|b|\}, \quad t_{0}=\sup \mathcal{T} .
$$

By (13), if $\lambda$ is sufficiently close to $\lambda_{2}$, then $t=0$ belongs to $\mathcal{T}$ so $\mathcal{T} \neq \emptyset$. By the definition of $\gamma, t_{0}$ cannot be infinite. But (12) implies that $t_{0}+1$ also belongs to $\mathcal{T}$, which is a contradiction.

Proposition 4.12. $\lambda_{3} \in \partial\left(-C_{R 2}\right) \cap\left(\widehat{\mathbb{C}} \backslash \mathrm{cl} \Lambda_{0}\right)$, where $-C_{R 2}$ is a regular component of $\Lambda_{\infty}$. Moreover, there exist $r_{0}>0$ and $0<\alpha<\pi / 2$ such that for $\lambda=\lambda_{3}+r e^{i \theta}$ and $0<r<r_{0}$,

- if $\alpha<\theta<2 \pi-\alpha$, then $\lambda \in-C_{R 2}$.

By symmetry, $-\lambda_{3}$ has analogous properties.

Proof. We only sketch the proof, since it proceeds as the previous one. If $\lambda \rightarrow \lambda_{3}$, then $f_{\lambda} \rightarrow g, g(z)=\frac{1+\sqrt{5}}{2} z(2-z)$ and $g$ is conformally conjugate to $z^{2}-1$. The point 0 is the landing point of the external ray in $B_{g}(\infty)$ with argument 0 . Moreover, there exists $0<\alpha<\pi / 2$ such that

$$
B_{g}(\infty) \supset\{z \in \mathbb{C} \backslash\{0\}: \alpha<\operatorname{Arg}(z)<2 \pi-\alpha\} .
$$

By (2) and (7), for $\lambda$ close to $\lambda_{3}$, we have

$$
\begin{gathered}
\frac{1}{b}=\frac{-15-7 \sqrt{5}}{8}\left(\lambda-\lambda_{3}\right)+\mathcal{O}\left(\left(\lambda-\lambda_{3}\right)^{2}\right), \\
\text { if } z=x \cdot \frac{1}{b}+\mathcal{O}\left(1 / b^{2}\right), \text { then } f_{\lambda}(z)=\frac{(1+\sqrt{5}) x^{2}}{x+1} \cdot \frac{1}{b}+\mathcal{O}\left(1 / b^{2}\right)
\end{gathered}
$$


and

$$
\text { if } z=x \cdot \frac{1}{b}+\mathcal{O}\left(1 / b^{2}\right) \text { for }|x|>\frac{\sqrt{5}}{5}+\varepsilon, \text { then }\left|f_{\lambda}(z)\right|>q|z| .
$$

Furthermore, by (3) and (7),

$$
u_{\lambda}=-2 \cdot \frac{1}{b}+\mathcal{O}\left(1 / b^{2}\right), \quad f_{\lambda}\left(u_{\lambda}\right)=-4(1+\sqrt{5}) \frac{1}{b}+\mathcal{O}\left(1 / b^{2}\right) .
$$

The rest of the proof is the same as for Proposition 4.11.

Proposition 4.13. $\lambda_{4} \in \partial C_{R 2} \cap \partial\left(-C_{R 2}\right)$ and $\lambda_{5} \in \partial C_{R 1} \cap \partial\left(-\bar{C}_{R 1}\right)$, where $C_{R 1}, C_{R 2}$ are regular components of $\Lambda_{0}$ and $-\bar{C}_{R 1},-C_{R 2}$ are regular components of $\Lambda_{\infty}$. Moreover, for every $\pi / 2<\theta<3 \pi / 2$ (resp. $-\pi / 2<$ $\theta<\pi / 2)$ there exists $r_{0}>0$ such that:

- if $\lambda=\lambda_{4}+r e^{i \theta}$ and $0<r<r_{0}$, then $\lambda \in C_{R 2}\left(\right.$ resp. $\left.\lambda \in-C_{R 2}\right)$,

- if $\lambda=\lambda_{5}+r e^{i \theta}$ and $0<r<r_{0}$, then $\lambda \in-\bar{C}_{R 1}$ (resp. $\left.\lambda \in C_{R 1}\right)$.

By symmetry, $-\lambda_{5}$ has analogous properties.

Proof. Consider $\lambda$ close to $\lambda_{4}=0$. Here $f_{\lambda} \rightarrow g$, where $g(z)=z^{2}$. Since by (7), $b-1=6 \lambda+\mathcal{O}\left(\lambda^{2}\right)$, we replace $\lambda$ close to 0 by $b$ close to 1 . As previously, define $\operatorname{Err}_{\lambda}(z)=f_{\lambda}(z)-g(z)$. By (2) and (7), we have

$$
\left|\operatorname{Err}_{\lambda}(z)\right|<c \frac{|z-1| \cdot|b-1|}{|(2-b) z-1|}
$$

provided $|z|<R$ for some large fixed $R$. Hence,

$$
\text { if } \quad c_{1}|b-1|<|z-1|<R, \quad \text { then }\left|\operatorname{Err}_{\lambda}(z)\right|<c_{2}|b-1|
$$

for some constant $c_{1}$ independent of $\lambda$ and $z$. Furthermore, by (2) and (7), for a fixed number $x \in \mathbb{C}$ and $\lambda$ near $\lambda_{4}$, if $z=1+x(b-1)+\mathcal{O}\left((b-1)^{2}\right)$, then

$$
f_{\lambda}(z)=1+\frac{2 x^{2}}{x-1}(b-1)+\mathcal{O}\left((b-1)^{2}\right) .
$$

Therefore, if $z=1+x(b-1)+\mathcal{O}\left((b-1)^{2}\right)$ for $|x|>1+\varepsilon$, then

$$
\left|f_{\lambda}(z)-1\right|>q|z-1|
$$

for $q>1$. Moreover, by (3) and (7),

$$
u_{\lambda}=1+2(b-1)+\mathcal{O}\left((b-1)^{2}\right), \quad f_{\lambda}\left(u_{\lambda}\right)=1+8(b-1)+\mathcal{O}\left((b-1)^{2}\right) .
$$

Let $\pi / 2<\theta<3 \pi / 2$ and take $\lambda$ close to $\lambda_{4}$ such that $\operatorname{Arg}(\lambda)=\theta$. Connect $u_{\lambda}$ to $f_{\lambda}\left(u_{\lambda}\right)$ by a straight line segment $I$. Then there exists $n_{0}$ such that for every $z \in I$,

$$
f_{\lambda}^{n_{0}}(z)=1+C(z)(b-1)+\mathcal{O}\left((b-1)^{2}\right),
$$


where $|C(z)|>M$ for a large $M$ and $|\operatorname{Im}(C(z))|<\delta$ for a small $\delta$. Hence for every $z \in I$,

$$
\theta-\varepsilon<\operatorname{Arg}\left(f_{\lambda}^{n_{0}}(z)-1\right)<\theta+\varepsilon
$$

for a small $\varepsilon$. Therefore, if we take $\lambda$ sufficiently close to $\lambda_{4}$, then for all $z \in I$,

$$
\left|f_{\lambda}^{n_{0}}(z)\right|<1-M^{\prime}|b-1|
$$

for a large $M^{\prime}$. By $(22)$,

$$
\operatorname{cl} f_{\lambda}\left(\mathbb{D}_{1-M^{\prime}|b-1|}\right) \subset \mathbb{D}_{1-M^{\prime}|b-1|},
$$

so $f_{\lambda}^{n_{0}}(I) \subset B_{f_{\lambda}}(0)$. This implies $u_{\lambda} \in B_{f_{\lambda}}(0)$.

We skip the proof of the remaining cases, since they are very similar to the previous ones.

Note that in Propositions 4.11-4.13 we have not proved which component is regular and which is exotic. Moreover, we have not proved that the components denoted by $C_{R 1}, C_{R 2}$ etc. in different propositions are the same. All these facts will be shown in Corollary 4.16.

4.3. Hyperbolic components in $\Lambda$. Now we prove that every regular component of $\Lambda_{0}, \Lambda_{\infty}$ is a topological disc and every exotic component is a punctured topological disc with a singular parameter as the isolated point of its boundary. Let $\zeta=0, \infty$. Denote by $\Phi_{\lambda}$ the Böttcher coordinates in the neighbourhood of $\zeta$. Recall that $\Phi_{\lambda}$ is a biholomorphic map from a neighbourhood $U$ of $\zeta$ onto some neighbourhood $V$ of 0 such that $\Phi_{\lambda}(\zeta)=0$ and the diagram

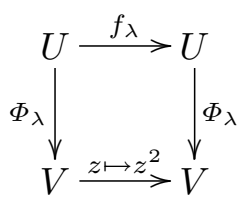

commutes (in the case when $u_{\lambda}=\zeta$, i.e. $\zeta$ is a double critical point, one should replace $z \mapsto z^{2}$ by $z \mapsto z^{3}$ ). If $\zeta=0$, then $\Phi_{\lambda}$ can be defined as

$$
\Phi_{\lambda}(z)=\lim _{n \rightarrow \infty} \sqrt[2^{n}]{f_{\lambda}^{n}(z)}
$$

for suitable branches of the root (for $\zeta=\infty$ one should take the inverse of this limit). The sequence is uniformly convergent in the neighbourhood of $\zeta$. If $\zeta$ is a simple critical point, then $\Phi_{\lambda}$ is uniquely determined and if $\zeta$ is a double critical point, then $\Phi_{\lambda}$ is determined uniquely up to multiplication by \pm 1 . Moreover, $\Phi_{\lambda}$ depends holomorphically on the map $f_{\lambda}$. If $u_{\lambda} \notin B(\zeta)$, then we can extend biholomorphically $\Phi_{\lambda}$ to $B(\zeta)$ by putting $\Phi_{\lambda}(z)=\sqrt[2^{n}]{\Phi_{\lambda}\left(f_{\lambda}^{n}(z)\right)}$ for the appropriate branch of the root. In this case $\Phi_{\lambda}$ is a Riemann mapping from $B(\zeta)$ onto the unit disc. If $u_{\lambda} \in B(\zeta)$, then we can proceed with the extension without difficulties until we meet $u_{\lambda}$. 
Strictly speaking, $\Phi_{\lambda}$ can be extended biholomorphically to some topological disc $\Omega_{\lambda}=\Phi_{\lambda}^{-1}\left(\mathbb{D}_{r}\right)$ for some $r<1$ such that $u_{\lambda} \in \partial \Omega_{\lambda}$.

Let $C$ be a component of $\Lambda_{\zeta}, \zeta=0, \infty$. By symmetry, we can consider only components of $\Lambda_{0}$. Assume first that $C$ is regular, i.e. for $f_{\lambda}, \lambda \in C$, the immediate basin $B(0)$ is simply connected. The standard way to parameterize a hyperbolic component is to map a point $\lambda \in C$ to $\Phi_{\lambda}\left(f_{\lambda}\left(u_{\lambda}\right)\right) \in \mathbb{D}$. However, since $C$ is regular, we can define $\Phi_{\lambda}\left(u_{\lambda}\right)$ and parameterize $C$ mapping $\lambda$ to $\Phi_{\lambda}\left(u_{\lambda}\right)$. In this way we obtain more information about the dynamics. This can be done since in this case the Böttcher coordinates $\Phi_{\lambda}$ extend diffeomorphically to $\mathrm{cl} \Omega_{\lambda}\left({ }^{1}\right)$. To see this it is enough to notice that if we define $\Gamma=\Phi_{\lambda}^{-1}\left(\left\{|z|=r_{0}\right\}\right)$ for $r_{0}$ such that $f_{\lambda}\left(u_{\lambda}\right) \in \Gamma$ (i.e. $\Gamma$ is the equipotential curve in $\Omega_{\lambda}$ containing $f_{\lambda}\left(u_{\lambda}\right)$ ), then $\partial \Omega_{\lambda}=f_{\lambda}^{-1}(\Gamma) \cap \operatorname{cl} \Omega_{\lambda}$ forms a Jordan curve (see Fig. 5).

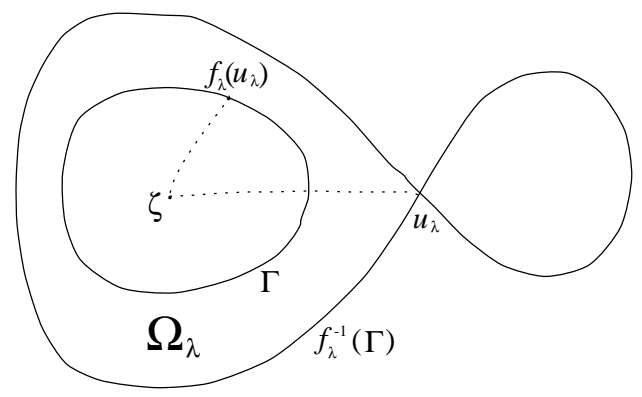

Fig. 5. The set $\Omega_{\lambda}$ in the regular case

Therefore, for every regular component $C$ of $\Lambda_{0}$ we can define

$$
\Psi: C \rightarrow \mathbb{D}, \quad \Psi(\lambda)=\Phi_{\lambda}\left(u_{\lambda}\right) .
$$

TheOREM 4.14. Every regular component $C$ is a topological disc and $\Psi: C \rightarrow \mathbb{D}$ is a surjective holomorphic mapping of degree two with one critical point $\lambda_{c} \in C$ such that $\Psi\left(\lambda_{c}\right)=0$.

Proof. The mapping $(z, \lambda) \mapsto \Phi_{\lambda}(z)$ is holomorphic for $z \in \Omega_{\lambda}$. Moreover, $\Psi(\lambda)=\Phi_{\lambda}\left(u_{\lambda}\right)$ can be defined as $\sqrt{\Phi_{\lambda}\left(f_{\lambda}\left(u_{\lambda}\right)\right)}$ for a branch of the square root depending continuously on $\lambda$. Hence $\Psi$ is holomorphic.

Now we show that $\Psi$ is a proper map onto $\mathbb{D}$. Take a sequence $\lambda_{n}$ converging to some $\lambda_{0} \in \partial C$. We now show that $\Psi\left(\lambda_{n}\right)$ converges to $\partial \mathbb{D}$. Suppose the converse. Taking a subsequence, we can assume $\Psi\left(\lambda_{n}\right) \rightarrow z_{0} \in \mathbb{D}$. Note that by Propositions $4.10-4.12$, we have $-\lambda_{1}, \lambda_{2}, \lambda_{3} \notin \mathrm{cl} \Lambda_{0}$, so $\lambda_{0} \neq-\lambda_{1}, \lambda_{2}, \lambda_{3}$. We also have $\lambda_{0} \neq \lambda_{1}$, because $\lambda_{1}$ is an isolated point of the boundary of an exotic component.

$\left({ }^{1}\right)$ This idea is due to Tan Lei. 
By (2), Lemma 2.2 and Corollary 4.1, the parameters $\pm \lambda_{1}, \lambda_{2}, \lambda_{3}$ are the only parameters where the basin of 0 degenerates. Hence for every $\lambda \in \widehat{\mathbb{C}} \backslash\left\{ \pm \lambda_{1}, \lambda_{2}, \lambda_{3}\right\}$, the map $\Phi_{\lambda}$ is defined in some neighbourhood $U_{\lambda}$ of 0. Moreover, $(\lambda, z) \mapsto \Phi_{\lambda}(z)$ is continuous and holomorphic for $\lambda \in$ $\widehat{\mathbb{C}} \backslash\left\{ \pm \lambda_{1}, \lambda_{2}, \lambda_{3}\right\}, z \in U_{\lambda}$.

Since $\lambda_{0} \neq \pm \lambda_{1}, \lambda_{2}, \lambda_{3}$, there exists $\delta>0$ and a small neighbourhood $\mathcal{U}$ of $\lambda_{0}$ such that for $\lambda \in \mathcal{U}$ the map $\Phi_{\lambda}$ is defined on $\mathbb{D}_{\delta}$. Hence there exists $\delta^{\prime}>0$ such that for $\lambda \in \mathcal{U}$ the map $\Phi_{\lambda}^{-1}$ is defined on $\mathbb{D}_{\delta^{\prime}}$ and $\Phi_{\lambda}^{-1}\left(\mathbb{D}_{\delta^{\prime}}\right) \subset \mathbb{D}_{\delta}$.

Of course, $\lambda_{n} \in \mathcal{U}$ for large $n$. As $\Psi\left(\lambda_{n}\right) \rightarrow z_{0} \in \mathbb{D}$, there exists a positive integer $k$ such that

$$
\left|\left(\Phi_{\lambda_{n}}\left(f_{\lambda_{n}}\left(u_{\lambda_{n}}\right)\right)\right)^{2^{k}}\right|<\delta^{\prime}
$$

for sufficiently large $n$. But $\left(\Phi_{\lambda_{n}}(z)\right)^{2^{k}}=\Phi_{\lambda_{n}}\left(f_{\lambda_{n}}^{k}(z)\right)$, so

$$
\left|\Phi_{\lambda_{n}}\left(f_{\lambda_{n}}^{k+1}\left(u_{\lambda_{n}}\right)\right)\right|<\delta^{\prime} .
$$

Thus,

$$
\left|f_{\lambda_{n}}^{k+1}\left(u_{\lambda_{n}}\right)\right|<\delta
$$

Note that by repeating the proofs of Propositions 4.11-4.12, one can easily show that if $\lambda_{n}$ is close enough to $-\lambda_{2}$ or $-\lambda_{3}$, then $f_{\lambda_{n}}^{k+1}\left(u_{\lambda_{n}}\right)$ is close to $\infty$ (cf. e.g. (11), (13)). Similarly, if $\lambda_{n}$ is close enough to $\lambda_{4}$ or $\pm \lambda_{5}$, then $f_{\lambda_{n}}^{k+1}\left(u_{\lambda_{n}}\right)$ is close to 1 (proof of Proposition 4.13). This implies that $\lambda_{0}=-\lambda_{2},-\lambda_{3}, \lambda_{4}, \pm \lambda_{5}$ contradicts (23). As already mentioned, $\lambda_{0}$ cannot be equal to $\pm \lambda_{1}, \lambda_{2}, \lambda_{3}$. We are left with the case $\lambda_{0} \in \Lambda$. But then by (23) and continuity, we have

$$
\left|f_{\lambda_{0}}^{k+1}\left(u_{\lambda_{0}}\right)\right| \leq \delta
$$

which means that for $f_{\lambda_{0}}$, the critical point $u_{\lambda_{0}}$ is in the basin of 0 . This clearly contradicts $\lambda_{0} \in \partial C$. In this way we have proved that $\Psi$ is a proper map from $C$ onto $\mathbb{D}$.

Now we show that $\Psi$ has no critical point at any $\lambda_{0} \in C$, provided $\Psi\left(\lambda_{0}\right) \neq 0$. Note that it is sufficient to show that $\lambda \mapsto(\Psi(\lambda))^{4}$ has no critical point at $\lambda_{0}$. For simplicity, define

$$
\varrho(\lambda)=(\Psi(\lambda))^{4}=\Phi_{\lambda}\left(f_{\lambda}^{2}\left(u_{\lambda}\right)\right) .
$$

Set also $\varrho_{0}=\varrho\left(\lambda_{0}\right)$. The proof is similar to the Douady-Hubbard-Sullivan proof of the fact that for $z^{2}+c$ each hyperbolic island in the Mandelbrot set corresponding to an attracting periodic orbit is biholomorphically parameterized by the multiplier of this orbit. To show that $\lambda \mapsto \varrho(\lambda)$ has no critical point at $\lambda_{0}$, we construct an inverse continuous mapping $\varrho \mapsto \lambda(\varrho)$ in the neighbourhood of $\varrho_{0}$.

Define

$$
A=\Phi_{\lambda_{0}}^{-1}\left(\left\{z: \sqrt{\left|\varrho_{0}\right|}-\varepsilon<|z|<\sqrt{\left|\varrho_{0}\right|}+\varepsilon\right\}\right)
$$


for a small $\varepsilon>0$. Then $A$ is a topological annulus in $\Omega_{\lambda_{0}}, f_{\lambda_{0}}\left(u_{\lambda_{0}}\right) \in A$, $u_{\lambda_{0}}, f_{\lambda_{0}}^{2}\left(u_{\lambda_{0}}\right) \notin A$ and $\left.f_{\lambda_{0}}\right|_{A}$ is a cover of degree two. Take $\varrho$ in a small neighbourhood of $\varrho_{0}$. It is easy to define a smooth cover $F_{\varrho}: \operatorname{cl} A \stackrel{\text { onto }}{\longrightarrow}$ $\operatorname{cl} f_{\lambda_{0}}(A)$ of degree two such that $F_{\varrho}=f_{\lambda_{0}}$ on $\partial A$ and $F_{\varrho}\left(f_{\lambda_{0}}\left(u_{\lambda_{0}}\right)\right)=$ $\Phi_{\lambda_{0}}^{-1}(\varrho)$. We also require that $F_{\varrho}$ depends continuously on $\varrho$ and $F_{\varrho_{0}}=$ $f_{\lambda_{0}} \mid \mathrm{cl} A$. Now we define

$$
g_{\varrho}= \begin{cases}f_{\lambda_{0}} & \text { on } \widehat{\mathbb{C}} \backslash A, \\ F_{\varrho} & \text { on } A\end{cases}
$$

By construction, $g_{\varrho}$ is a branched covering of $\widehat{\mathbb{C}}$, holomorphic outside cl $A$. Moreover, $g_{\varrho}$ depends continuously on $\varrho$ and $g_{\varrho_{0}}=f_{\lambda_{0}}$. Let $\mu_{0}$ be the standard conformal structure on $\widehat{\mathbb{C}}$. Define a continuous family of conformal structures $\mu_{\varrho}$ :

$$
\mu_{\varrho}= \begin{cases}\mu_{0} & \text { on } \Phi_{\lambda_{0}}^{-1}\left(\mathbb{D} \sqrt{\left|\varrho_{0}\right|}-\varepsilon\right) \\ \left(g_{\varrho}^{n}\right)^{*}\left(\mu_{\varrho}\right) & \text { on } g_{\varrho}^{-n}\left(\Phi_{\lambda_{0}}^{-1}\left(\mathbb{D} \sqrt{\left|\varrho_{0}\right|}-\varepsilon\right)\right), \\ \mu_{0} & \text { else. }\end{cases}
$$

Then $g_{\varrho}$ preserves $\mu_{\varrho}$ almost everywhere on $\widehat{\mathbb{C}}$. Moreover, for any point in $\widehat{\mathbb{C}}$ its forward trajectory under $g_{\varrho}$ hits at most once the annulus $A$, where $g_{\varrho}$ is not holomorphic. Since $g_{\varrho}$ is smooth on $\operatorname{cl} A, \mu_{\varrho}$ is changed on $A$ only by a bounded factor. This implies that $\mu_{\varrho}$ is bounded. Using the measurable Riemann theorem we can integrate $\mu_{\varrho}$ to obtain a continuous family of quasiconformal homeomorphisms $H_{\varrho}$ such that $H_{\varrho}(0)=0$, $H_{\varrho}(1)=1, H_{\varrho}(\infty)=\infty$ and $H_{\varrho} g_{\varrho} H_{\varrho}^{-1}$ is a rational map. By construction, $H_{\varrho} g_{\varrho} H_{\varrho}^{-1}$ has supersinks at 0 and $\infty$ and 1 is a critical point of period two. Hence $H_{\varrho} g_{\varrho} H_{\varrho}^{-1}=f_{\lambda(\varrho)}$ for some $\lambda(\varrho)$. Since $\varrho \mapsto f_{\lambda(\varrho)}$ is continuous and $f_{\lambda}(1)=(1+\lambda) /(1-\lambda)$, the map $\varrho \mapsto \lambda(\varrho)$ is continuous. By construction, $\lambda\left(\varrho_{0}\right)=\lambda_{0}$ and $\lambda(\varrho) \in C$.

To show that $\varrho \mapsto \lambda(\varrho)$ is the inverse mapping of $\lambda \mapsto \varrho(\lambda)$, note that since a conjugation maps critical points to critical points, we have $u_{\lambda(\varrho)}=$ $H_{\varrho}\left(u_{\lambda_{0}}\right)$, so

$$
f_{\lambda(\varrho)}^{2}\left(u_{\lambda(\varrho)}\right)=H_{\varrho}\left(g_{\varrho}^{2}\left(u_{\lambda_{0}}\right)\right) .
$$

Moreover, the map $\Phi_{\lambda_{0}} \circ H_{\varrho}^{-1}$ conformally conjugates $f_{\lambda(\varrho)}$ to $z \mapsto z^{2}$ on $H_{\varrho}\left(\Phi_{\lambda_{0}}^{-1}\left(\mathbb{D} \sqrt{\left|\varrho_{0}\right|}-\varepsilon\right)\right)$ and $f_{\lambda(\varrho)}^{2}\left(u_{\lambda(\varrho)}\right) \in H_{\varrho}\left(\Phi_{\lambda_{0}}^{-1}\left(\mathbb{D} \sqrt{\left|\varrho_{0}\right|}-\varepsilon\right)\right)$. Hence, by the uniqueness of the Böttcher coordinates, we have

$$
\Phi_{\lambda(\varrho)}\left(f_{\lambda(\varrho)}^{2}\left(u_{\lambda(\varrho)}\right)\right)=\Phi_{\lambda_{0}}\left(H_{\varrho}^{-1}\left(f_{\lambda(\varrho)}^{2}\left(u_{\lambda(\varrho)}\right)\right)\right) .
$$

By definition,

$$
\varrho(\lambda(\varrho))=\Phi_{\lambda(\varrho)}\left(f_{\lambda(\varrho)}^{2}\left(u_{\lambda(\varrho)}\right)\right),
$$


so by $(25),(24)$ and the definition of $g_{\varrho}$ we obtain

$$
\varrho(\lambda(\varrho))=\Phi_{\lambda_{0}}\left(g_{\varrho}^{2}\left(u_{\lambda_{0}}\right)\right)=\varrho .
$$

Hence $\varrho \mapsto \lambda(\varrho)$ is the inverse mapping of $\lambda \mapsto \varrho(\lambda)$, which ends the proof that $\Psi$ has no critical point at $\lambda_{0}$ provided $\Psi\left(\lambda_{0}\right) \neq 0$.

By the Riemann-Hurwitz formula, this implies that $C$ is a topological disc and there exists a unique point $\lambda_{c} \in C$ such that $\Psi\left(\lambda_{c}\right)=0$. Now we show that $\lambda_{c}$ is a simple $\Psi$-critical point. By (8),

$$
f_{\lambda}(z)=z^{2} h_{\lambda}(z)
$$

for

$$
h_{\lambda}(z)=\frac{\lambda+1}{\lambda-1} \cdot \frac{(\lambda-1)\left(\lambda^{2}-4 \lambda-1\right) z-\left(3 \lambda^{3}-5 \lambda^{2}+9 \lambda+1\right)}{\left(3 \lambda^{3}+5 \lambda^{2}+9 \lambda-1\right) z-(\lambda+1)\left(\lambda^{2}+4 \lambda-1\right)} .
$$

The condition $\Psi\left(\lambda_{c}\right)=0$ means that $u_{\lambda_{c}}=0$, so by Proposition $4.8, \lambda_{c}$ is a simple root of the polynomial $3 \lambda^{3}-5 \lambda^{2}+9 \lambda+1$. This together with (26) implies that for every constant $c>0$ there exists a neighbourhood $U$ of $\lambda_{c}$ and constants $c_{1}, c_{2}>0$ such that for $\lambda \in U$ and every $z$,

$$
\text { if }|z|<c\left|\lambda-\lambda_{c}\right|^{2} \text {, then } c_{1}\left|\lambda-\lambda_{c}\right|<\left|h_{\lambda}(z)\right|<c_{2}\left|\lambda-\lambda_{c}\right| .
$$

Then by (26),

$$
c_{1}\left|\lambda-\lambda_{c}\right| \cdot|z|^{2}<\left|f_{\lambda}(z)\right|<c_{2}\left|\lambda-\lambda_{c}\right| \cdot|z|^{2}
$$

and induction gives

$$
\left(c_{1}\left|\lambda-\lambda_{c}\right|\right)^{2^{n}-1}|z|^{2^{n}}<\left|f_{\lambda}^{n}(z)\right|<\left(c_{2}\left|\lambda-\lambda_{c}\right|\right)^{2^{n}-1}|z|^{2^{n}} .
$$

By (9), there exist constants $c_{3}, c_{4}>0$ such that for $\lambda$ close to $\lambda_{c}$,

$$
c_{3}\left|\lambda-\lambda_{c}\right|^{3}<\left|f_{\lambda}\left(u_{\lambda}\right)\right|<c_{4}\left|\lambda-\lambda_{c}\right|^{3},
$$

so the assumption of $(27)$ holds for $z=f_{\lambda}\left(u_{\lambda}\right)$. Therefore,

$$
c_{1}^{-1}\left(c_{1} c_{3}\right)^{2^{n-1}}\left|\lambda-\lambda_{c}\right|^{2^{n+1}-1}<\left|f_{\lambda}^{n}\left(u_{\lambda}\right)\right|<c_{2}^{-1}\left(c_{2} c_{4}\right)^{2^{n-1}}\left|\lambda-\lambda_{c}\right|^{2^{n+1}-1}
$$

for $\lambda$ in some neighbourhood $U$ of $\lambda_{c}$. Recall that in the regular case

$$
\Psi(\lambda)=\lim _{n \rightarrow \infty} \sqrt[2^{n}]{f_{\lambda}^{n}\left(u_{\lambda}\right)}
$$

Hence there exists a constant $c_{5}>0$ such that for every $\lambda \in U$,

$$
c_{5}^{-1}\left|\lambda-\lambda_{c}\right|^{2}<|\Psi(\lambda)|<c_{5}\left|\lambda-\lambda_{c}\right|^{2} .
$$

This implies that $\lambda_{c}$ is a simple $\Psi$-critical point.

Consider now the case when $C$ is an exotic component. Then the boundary of $\Omega_{\lambda}$ is not a Jordan curve and we cannot extend $\Phi_{\lambda}$ to $\mathrm{cl} \Omega_{\lambda}$ (the situation is the same as for the basin of infinity for $\left.z^{2}+c, c \notin M\right)$. Hence, for every exotic component $C$ we define

$$
\Psi: C \rightarrow \mathbb{D}, \quad \Psi(\lambda)=\Phi_{\lambda}\left(f_{\lambda}\left(u_{\lambda}\right)\right)
$$


$\Psi$ is holomorphic, since $(z, \lambda) \mapsto \Phi_{\lambda}(z)$ is holomorphic for $z \in \Omega_{\lambda}$. If $C$ is the exotic component for which $\lambda_{1}$ is an isolated point of the boundary (see Proposition 4.10), we extend $\Psi$ holomorphically to $C \cup\left\{\lambda_{1}\right\}$. Write for simplicity $\widetilde{C}=C \cup\left\{\lambda_{1}\right\}$ if $\lambda_{1} \in \partial C$ and $\widetilde{C}=C$ otherwise (in fact, the proof will show that the second case does not occur, but a priori we do not know this).

TheOREM 4.15. For every exotic component $C, \Psi: \widetilde{C} \rightarrow \mathbb{D}$ is a surjective holomorphic mapping of degree two with one simple critical point $\lambda_{c}$ such that $\Psi\left(\lambda_{c}\right)=0$.

Proof. The proof that $\Psi$ is a proper holomorphic map onto $\mathbb{D}$ is the same as for the regular case. The surjectivity of $\Psi$ implies that there exists exactly one exotic component $C_{E}$ of $\Lambda_{0}, \lambda_{c}=\lambda_{1}$ is an isolated point of $\partial C_{E}$ and $\Psi\left(\lambda_{1}\right)=0$. The same proof as for the regular case shows that $\Psi$ has no critical points in $C_{E}=\widetilde{C} \backslash\left\{\lambda_{1}\right\}$. Therefore, $C_{E}$ is a punctured topological disc.

To show that $\lambda_{c}=\lambda_{1}=-1$ is a simple $\Psi$-critical point, consider the behaviour of $\Psi$ in the neighbourhood of -1 . We proceed as previously. By (26), one can easily compute that for every constant $c>0$ there exists a neighbourhood $U$ of -1 such that for $\lambda \in U$,

$$
\text { if }|z|<c|\lambda+1|^{2} \text {, then } 1<\left|h_{\lambda}(z)\right|<3 .
$$

This gives

$$
|z|^{2}<\left|f_{\lambda}(z)\right|<3|z|^{2}
$$

and by induction,

$$
|z|^{2^{n}}<\left|f_{\lambda}^{n}(z)\right|<3^{2^{n}-1}|z|^{2^{n}}
$$

Using (9), we check that $f_{\lambda}\left(u_{\lambda}\right)=2(\lambda+1)^{2}+\mathcal{O}\left((\lambda+1)^{3}\right)$ for $\lambda \rightarrow-1$. Therefore for $\lambda$ near -1 ,

$$
|\lambda+1|^{2}<\left|f_{\lambda}\left(u_{\lambda}\right)\right|<3|\lambda+1|^{2},
$$

so

$$
|\lambda+1|^{2^{n}}<\left|f_{\lambda}^{n}\left(u_{\lambda}\right)\right|<3^{2^{n}-1}|\lambda+1|^{2^{n}} .
$$

Recall that in the exotic case

$$
\Psi(\lambda)=\lim _{n \rightarrow \infty} \sqrt[2^{n}]{f_{\lambda}^{n}\left(f_{\lambda}\left(u_{\lambda}\right)\right)}=\lim _{n \rightarrow \infty} \sqrt[2^{n-1}]{f_{\lambda}^{n}\left(u_{\lambda}\right)} .
$$

Hence, for every $\lambda \in U$,

$$
|\lambda+1|^{2}<|\Psi(\lambda)|<9|\lambda+1|^{2} .
$$

This implies that $\lambda_{1}=-1$ is a simple critical point for $\Psi$.

Corollary 4.16. $\Lambda_{0}$ consists of three regular components: $C_{R 1}, \bar{C}_{R 1}$, $C_{R 2}$, and one exotic, $C_{E}$. Moreover, 
- $C_{R 1}$ is a topological disc in the right upper quarter of the plane,

- $\lambda_{7} \in C_{R 1}$ and $-\lambda_{2}, \lambda_{5} \in \partial C_{R 1}$,

- $\bar{C}_{R 1}$ is a topological disc in the right lower quarter of the plane,

- $\bar{\lambda}_{7} \in \bar{C}_{R 1}$ and $-\lambda_{2},-\lambda_{5} \in \partial \bar{C}_{R 1}$,

- $C_{R 2}$ is a topological disc in the left half plane, and $-\lambda_{3}, \lambda_{4} \in \partial C_{R 2}$,

- $C_{E} \cup\left\{\lambda_{1}\right\}$ is a topological disc in the left half plane.

By symmetry, $\Lambda_{\infty}$ has analogous properties.

Pro of. By Proposition 4.8, for $\Lambda_{0}$ there are exactly four parameters $\lambda$ such that $\Psi(\lambda)=0: \lambda_{1}, \lambda_{6}, \lambda_{7}$ and $\bar{\lambda}_{7}$. In Theorems 4.14 and 4.15 we have shown that each of these points is a "centre point" of a component of $\Lambda_{0}$. Hence there are exactly four components of $\Lambda_{0}$ : three regular and one exotic; moreover, the regular ones are topological discs and the exotic one is a punctured topological disc. By Proposition $4.8, \lambda_{6}, \lambda_{7}, \bar{\lambda}_{7}$ lie in regular components. Let $C_{R 1}$ be the component containing $\lambda_{7}, \bar{C}_{R 1}$ be the component containing $\bar{\lambda}_{7}$ and $C_{R 2}$ be the component containing $\lambda_{6}$. Note that by (10), the imaginary axis is disjoint from $\Lambda_{0}$. Moreover, $\operatorname{Re}\left(\lambda_{1}\right), \operatorname{Re}\left(\lambda_{6}\right)<0$ and $\operatorname{Re}\left(\lambda_{7}\right), \operatorname{Im}\left(\lambda_{7}\right)>0$. Hence (10) implies that the right half of the real axis is disjoint from $\Lambda_{0}$. Thus $C_{R 1}$ lies in the right upper quarter of the plane, $\bar{C}_{R 1}$ in the lower right quarter and $C_{R 2}$ in the left half plane. Denote by $C_{E}$ the unique exotic component. Then by Proposition 4.10, $\lambda_{1}$ is an isolated point in the boundary of $C_{E}$ so $C_{E}$ lies in the left half plane.

By Propositions 4.11 and $4.13,-\lambda_{2}$ and $\lambda_{5}$ are in the boundaries of components of $\Lambda_{0}$ which are contained in the right upper quarter of the plane, hence they must be in $\partial C_{R 1}$. Similarly, $-\lambda_{2}, \lambda_{5} \in \partial \bar{C}_{R 1}$.

Consider now the components of $\Lambda_{0}$ in the left half plane. By Propositions 4.12 and $4.13,-\lambda_{3}$ and $\lambda_{4}$ are in the boundaries of components of $\Lambda_{0}$ in the left half plane, and the points from the real axis slightly greater than $-\lambda_{3}$ or slightly less than $\lambda_{4}$ do not belong to $\Lambda_{0}$. Knowing that $C_{R 2}$ and $C_{E} \cup\left\{\lambda_{1}\right\}$ are topological discs symmetric with respect to the real axis and $\lambda_{1}<-\lambda_{3}<\lambda_{6}<\lambda_{4}$ one can easily conclude that $-\lambda_{3}, \lambda_{4} \in \partial C_{R 2}$.

The above corollary ends the proof of the classification of the components of $\Lambda_{0}$ and $\Lambda_{\infty}$ stated in Theorem 4.9. In the same way one can deal with the components of $\Lambda_{1}, \Lambda_{f_{\lambda}(1)}$. We omit the details.

Definition 4.17. Let $C$ be a component of $\Lambda_{\zeta}$ for $\zeta=0, \infty$. By the previous theorems, we can define internal rays in $C$ setting

$$
\mathcal{R}_{C}(\theta)=\Psi^{-1}\left(\left\{r e^{i \theta}: 0 \leq r<1\right\}\right) .
$$

Note that for given $\theta$ there are two internal rays in $C$ corresponding to $\theta$. In particular, we will prove that two 0-rays in $C_{R 1}$ end at parabolic maps connected with Newton's method (see Fig. 2). The same holds for $C_{E}$. 
4.4. Combinatorics of Newton maps. Let $N$ be a cubic Newton map. By a conformal change of coordinates, we can assume that $N \in \mathcal{F}$ and $N$ has supersinks at $0, u, \infty$. First we consider the general case, i.e. we do not assume that $N \in \mathcal{F}_{2}$. Denote by $B(\zeta)$ the immediate basin of attraction of $\zeta$ for $\zeta=0, u, \infty$. In the entire subsection we assume that the "free" critical point 1 is not in $B(0) \cup B(u) \cup B(\infty)$. Then $B(\zeta)$ is simply connected and $N$ on $B(\zeta)$ is conformally conjugate to $z \mapsto z^{2}$. (Recall that the Julia set for any Newton map is connected - see [Sh].)

Definition 4.18. Let $R_{\zeta}(\theta)$ be the internal ray in $B(\zeta)$ with angle $\theta \in$ $[0,1]$, i.e. $R_{\zeta}(\theta)=\Phi_{\zeta}^{-1}\left(\left\{r e^{2 \pi i \theta}: r<1\right\}\right)$, where $\Phi_{\zeta}: B(\zeta) \rightarrow \mathbb{D}$ are the Böttcher coordinates in $B(\zeta)$.

As $N$ has degree two on $B(\zeta)$, there exists exactly one component of $N^{-1}(B(\zeta))$ different from $B(\zeta)$. Denote this component by $\widetilde{B}(\zeta)$. Note that $N: \widetilde{B}(\zeta) \rightarrow B(\zeta)$ is biholomorphic. Let $\widetilde{R}_{\zeta}(\theta)=N^{-1}\left(R_{\zeta}(\theta)\right) \cap \widetilde{B}(\zeta)$.

Finally, for $\zeta=0,1, u, \infty$ define $l_{\zeta}(\theta)$ (resp. $\left.\widetilde{l}_{\zeta}(\theta)\right)$ to be the landing point of $R_{\zeta}(\theta)$ (resp. $\widetilde{R}_{\zeta}(\theta)$ ).

REMARK. In general, the rays have well-defined landing points only when the boundary is locally connected. However, according to the result proved in [Ro], for all cubic Newton maps the boundaries of the immediate basins of attractions to supersinks are locally connected.

Now we look at the combinatorics of $N$. We use the results proved in $[\mathrm{He}]$ and $[\mathrm{Ta}]$.

The map $N$ has four fixed points, so there exists exactly one fixed point $p$ which is not critical. Therefore, we have $p=l_{\infty}(0)=l_{0}(0)=l_{u}(0)$. Permuting $0, u, \infty$ we can assume that $R_{0}(0), R_{u}(0), R_{\infty}(0)$ are situated around $p$ in counter-clockwise order. Consider $N^{-1}(p) \backslash\{p\}$. It consists of two points: $p_{1}$ and $p_{2}$. On the other hand, it must contain the landing points of $R_{0}(1 / 2), R_{u}(1 / 2), R_{\infty}(1 / 2)$ and the landing points of $\widetilde{R}_{\infty}(0), \widetilde{R}_{0}(0), \widetilde{R}_{u}(0)$. Hence two of the points $l_{0}(1 / 2), l_{u}(1 / 2), l_{\infty}(1 / 2)$ must coincide. Moreover, the third one cannot coincide with them because in that case $N$ would change the cyclic order of the internal rays passing from $l_{0}(1 / 2)$ to $p$. Permuting $0, u, \infty$ once more, we can assume that $l_{u}(1 / 2)=l_{\infty}(1 / 2)=p_{1}$. This implies $l_{u}(1 / 2)=l_{\infty}(1 / 2)=\widetilde{l}_{0}(0)=p_{1}$ and $l_{0}(1 / 2)=\widetilde{l}_{u}(0)=\widetilde{l}_{\infty}(0)=p_{2}$. See $[\mathrm{He}]$ for details. According to $[\mathrm{Ta}]$ and $[\mathrm{Ro}]$, any two different internal rays in $B(\zeta)$ have distinct landing points (the boundary of $B(\zeta)$ is a Jordan curve). Moreover,

$$
\partial B(u) \cap \partial B(\infty)=\left\{z: z=l_{u}(\theta)=l_{\infty}(1-\theta) \text { for some } \theta \in[0,1]\right\}
$$

is a Cantor set, forward invariant under $N$. It contains the sequence $a_{n} \rightarrow p$, where $a_{n}=l_{u}\left(1-1 / 2^{n}\right)=l_{\infty}\left(1 / 2^{n}\right)$ for all positive integers $n$. On the 
other hand, there exists an angle $0<\alpha \leq 1 / 2$ (Head's angle) such that $\alpha=\inf \left\{\theta>0: l_{u}(\theta)=l_{\infty}(1-\theta)\right\}$. Moreover,

$$
\partial B(u) \cap \partial B(\infty)=\left\{l_{u}(\theta): \theta \in T_{N}\right\},
$$

where

$$
T_{N}=\left\{\theta: 2^{n} \theta \bmod 1 \in[\alpha, 1] \text { for every } n \geq 0\right\}
$$

and

$$
\begin{aligned}
\partial B(u) \cap \partial \widetilde{B}(\infty) & =\left\{z: z=l_{u}(\theta)=\widetilde{l}_{\infty}(1-2 \theta) \text { for some } \theta \in[0,1]\right\} \\
& =\left\{l_{u}(\theta / 2): \theta \in T_{N} \cap[\alpha, 2 \alpha]\right\} .
\end{aligned}
$$

The set $\partial B(\infty) \cap \partial \widetilde{B}(u)$ can be described in a similar way. Moreover,

$$
\begin{aligned}
\partial \widetilde{B}(u) \cap \partial \widetilde{B}(\infty) & =\left\{z: z=\widetilde{l}_{u}(\theta)=\widetilde{l}_{\infty}(1-\theta) \text { for some } \theta \in[0,1]\right\} \\
& =\left\{\widetilde{l}_{u}(\theta): \theta \in T_{N} \cap[2 \alpha, 1]\right\} .
\end{aligned}
$$

Furthermore, $\partial B(0) \cap(\partial B(u) \cup \partial B(\infty))=\{p\}$.

Let

$$
\beta=\inf \left\{\theta \in[0,1]: l_{u}(\omega) \notin \partial \widetilde{B}(\infty) \text { for every } \omega \in[\theta, 1]\right\} .
$$

The following facts were proved in $[\mathrm{He}]$ (see also $[\mathrm{Ta}]$ ):

Lemma 4.19. For $N$ as above, the critical point 1 is not equal to $l_{u}(\alpha)$ if and only if $\alpha$ is periodic mod 1 . Moreover, if $\alpha=m /\left(2^{k}-1\right)$ for some positive integers $m, k$, then $\beta=m / 2^{k}$ and there exists a topological disc $U$ such that $\operatorname{cl} U \subset N^{k}(U)$ and

$$
\left.N^{k}\right|_{U}: U \rightarrow N^{k}(U)
$$

is a polynomial-like mapping of degree two. Furthermore, $U$ contains a set $X$ bounded by the following four simple curves: $\left\{l_{u}(\theta): \theta \in[\beta, \alpha]\right\},\left\{l_{\infty}(\theta)\right.$ : $\theta \in[1-\alpha, 1-\beta]\},\left\{\widetilde{l}_{u}(\theta): \theta \in[2 \beta, 2 \alpha]\right\},\left\{\widetilde{l}_{\infty}(\theta): \theta \in[1-2 \alpha, 1-2 \beta]\right\}$ and the critical point 1 lies in $\mathrm{cl} X$. In particular, the landing point $l_{u}(\alpha)=l_{\infty}(\alpha)$ is a fixed point for $\left.N^{k}\right|_{U}$.

The set $U$ can be constructed as a domain with boundary consisting of parts of rays and equipotentials in $B(u), \widetilde{B}(u), B(\infty)$ and $\widetilde{B}(\infty)$.

Note that since $2 \beta>\alpha$, we have $2 \beta \in T_{N}$, so $l_{u}(2 \beta)=l_{\infty}(1-2 \beta)$. Moreover, the definition of $\alpha$ easily implies that for $j=0, \ldots, k-1,2^{j} \beta \neq$ $0 \bmod 1$, so $2^{k-1} \beta=1 / 2 \bmod 1$ and $2^{j} \beta \neq 1 / 2 \bmod 1$ for $j=0, \ldots, k-2$.

Assume now that $N$ is in $\mathcal{F}_{2}$. As mentioned in Section 3, this condition is equivalent to $a=((b-2) /(2 b-1))^{2}$, which gives $9 \lambda^{6}+49 \lambda^{4}-13 \lambda^{2}+19=0$. Hence there are six such maps in $\mathcal{F}_{2}: \pm N_{1}, \pm \bar{N}_{1}$ and $\pm N_{2}$, where $N_{1}=f_{\lambda}$ for $\lambda \approx 0.6175+0.4734 i$ and $N_{2}=f_{\lambda}$ for $\lambda \approx 2.3998 i$ (see Fig. 2). Note that for $N \in \mathcal{F}_{2}$, Head's angle $\alpha$ is equal to $1 / 3$. 


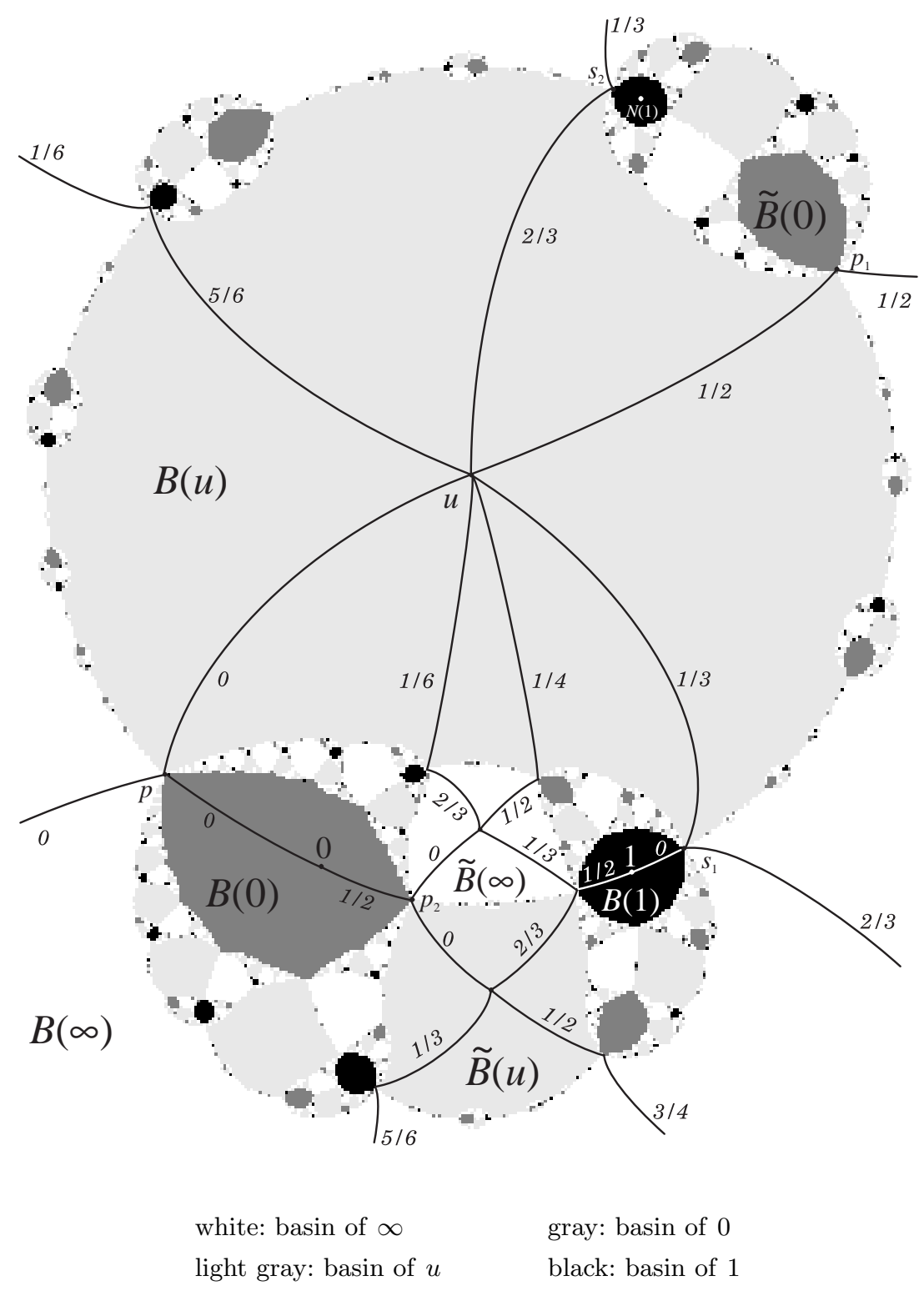

Fig. 6. The combinatorics of a Newton map of degree three with supersinks $0, u, \infty$ and the fourth critical point 1 of period two

Let $B(1)$ be the immediate basin of attraction of the periodic point 1 and define $R_{1}(\theta)=\Phi_{1}^{-1}\left(\left\{r e^{2 \pi i \theta}: r<1\right\}\right)$, where $\Phi_{1}: B(1) \rightarrow \mathbb{D}$ conjugates $N^{2}$ to $z \mapsto z^{2}$. As previously, denote by $l_{1}(\theta)$ the landing point of $R_{1}(\theta)$.

Consider periodic orbits of $N$ of length two. There are three such orbits (there are 10 fixed points of $N^{2}$ and 4 fixed points of $N$ ). The first orbit 
consists of 1 and $N(1)$, and the second of $l_{0}(1 / 3)$ and $l_{0}(2 / 3)$. Note that both these orbits are disjoint from $\partial B(u) \cup \partial B(\infty)$. Hence the third orbit consists of $l_{u}(1 / 3)=l_{\infty}(2 / 3)=s_{1}$ and $l_{u}(2 / 3)=l_{\infty}(1 / 3)=s_{2}$ for some $s_{1}, s_{2}$. The landing point of $R_{1}(0)$ is periodic with period two, so it must be equal to one of the points $s_{1}, s_{2}$. Consider $\widehat{\mathbb{C}} \backslash\left(\operatorname{cl} R_{\infty}(0) \cup \operatorname{cl} R_{u}(0) \cup\right.$ $\left.\operatorname{cl} R_{u}(1 / 2) \cup \operatorname{cl} R_{\infty}(1 / 2)\right)$. It has two components: $C_{1}$ containing $B(0)$ and $s_{1}$, and $C_{2}$ containing $\widetilde{B}(0)$ and $s_{2}$. We have $N\left(C_{2}\right)=\widehat{\mathbb{C}} \backslash\left(\operatorname{cl} R_{\infty}(0) \cup \operatorname{cl} R_{u}(0)\right)$ and $N$ has degree 1 on $C_{2}$. Hence there are no critical points in $C_{2}$, so $1 \in C_{1}$ and the landing point of $R_{1}(0)$ is equal to $s_{1}$. Note that $N^{-1}\left(s_{2}\right)=$ $\left\{s_{1}, l_{1}(1 / 2), l_{\infty}(1 / 6)\right\}$ and only the second point lies outside $\partial B(u) \cap \partial B(\infty)$. Therefore $l_{1}(1 / 2)=\widetilde{l}_{u}(2 / 3)=\widetilde{l}_{\infty}(1 / 3)$. See Fig. 6 .

4.5. Parabolic bifurcation in $\mathcal{F}_{2}$. Now we prove the existence of a parabolic bifurcation in $\mathcal{F}_{2}$. Starting from a Newton map and changing continuously the parameter $\lambda$, we arrive at a parabolic map which lies in the intersection of the boundary of an exotic and a non-exotic component of $\Lambda_{\zeta}$, $\zeta=0, \infty$.

TheOREM 4.20. Let $N$ be a Newton map in $\mathcal{F}_{2}$ such that $\partial B(u) \cap \partial B(\infty)$ is infinite. Then there exist simple open curves $\gamma, \gamma_{0}, \gamma_{\infty}:[0,1] \rightarrow \Lambda$ such that:

(a) $f_{\gamma(0)}=N$ and for every $t \in(0,1)$, the map $f_{\gamma(t)}$ has an attracting fixed point $\xi_{t} \neq 0, \infty$ with multiplier $t$.

(b) $\gamma(1)=\gamma_{0}(0)=\gamma_{\infty}(0)$ and the map $P=f_{\gamma(1)}$ has a parabolic fixed point with multiplier 1.

(c) For every $t \in(0,1]$, for the map $f_{\gamma_{0}(t)}$ the critical point $u$ is in the immediate basin $B(0)$, and $B(0)$ is not exotic.

(d) For every $t \in(0,1]$, for the map $f_{\gamma_{\infty}(t)}$ the critical point $u$ is in the immediate basin $B(\infty)$, and $B(\infty)$ is exotic.

See Figs. 7 and 8.

Proof. 1. The existence of $\gamma$. Take $\lambda_{N}$ such that $N=f_{\lambda_{N}}$. It is obvious that after a small perturbation of $\lambda_{N}$ we obtain a map with an attracting fixed point $\xi_{\lambda}$ close to $u_{\lambda}$ such that $u_{\lambda} \in B\left(\xi_{\lambda}\right)$. Consider the component $U \ni \lambda_{N}$ of the set of parameters $\lambda \in \widehat{\mathbb{C}}$ such that for the map $f_{\lambda}$ there exists an attracting fixed point $\xi_{\lambda} \neq 0, \infty$ and $u_{\lambda} \in B\left(\xi_{\lambda}\right)$. We claim that there are no singular parameters in $U$. To see this, suppose that $\lambda_{0} \in U$ is a singular parameter. By uniform convergence (see Corollary 4.1), there exists an open neighbourhood of $\lambda_{0}$ contained in $U$. But this is impossible, by the characteristics of the singular parameters (see Subsection 4.2). If $\lambda \in \partial U \cap \Lambda$, then $f_{\lambda}$ has an indifferent fixed point (i.e. the multiplier has absolute value 1 ). Thus $\partial U \cap \Lambda$ is piecewise analytic, with cusps at points where $f_{\lambda}$ has a fixed point with multiplier 1 . 


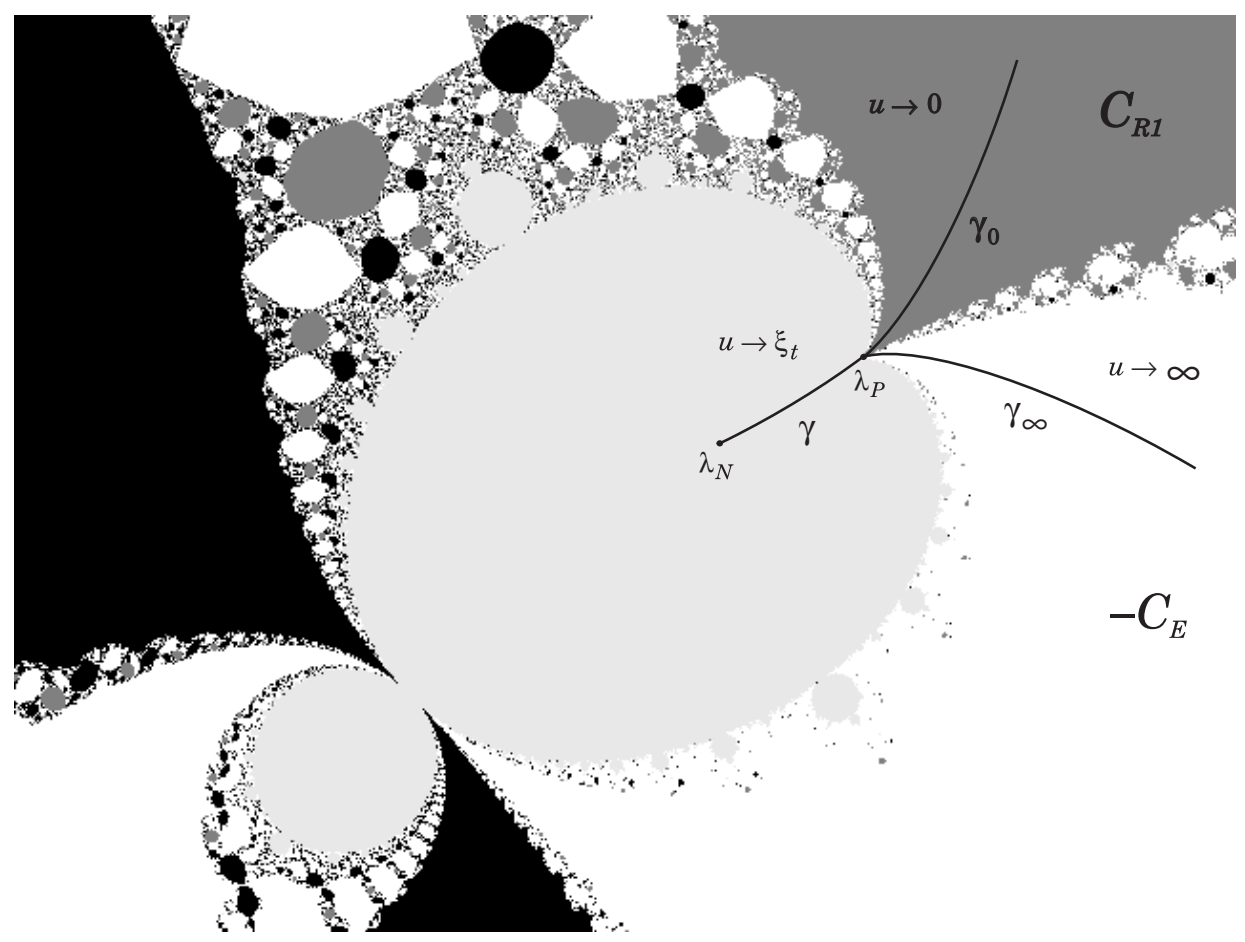

Fig. 7. Bifurcation at the parabolic map

To complete the proof of the existence of $\gamma$ we need to show that there are no singular parameters in $\partial U$. This will be done in the general case in the second part of the work. Here we show (for example) that $\lambda_{2} \notin \partial U$. Recall that $\lambda_{2}=-2-\sqrt{5}$ and Corollary 4.1 implies that if $\lambda \rightarrow \lambda_{2}$ then $f_{\lambda} \rightarrow g$ uniformly outside a small neighbourhood of 0 , where $g(z)=((1-$ $\sqrt{5}) / 2) z(2-z)$. The fixed points of $g$ are 0 (with multiplier $1-\sqrt{5}$ ) and $(5+\sqrt{5}) / 2$ (with multiplier $1+\sqrt{5}$ ). The fixed points of $f_{\lambda}$ are $0, \infty$ and two others - denote them by $\xi_{\lambda}, \widetilde{\xi}_{\lambda}$. By the implicit function theorem and uniform convergence, if $\lambda \rightarrow \lambda_{2}$ then one of these points (say $\xi_{\lambda}$ ) tends to $(5+\sqrt{5}) / 2$ and $f_{\lambda}^{\prime}\left(\xi_{\lambda}\right)$ tends to $1+\sqrt{5}>1$, so $\xi_{\lambda}$ is not attracting. Moreover, by the holomorphic fixed point formula (see e.g. [Mi1]), we have

$$
\frac{1}{1-f_{\lambda}^{\prime}\left(\xi_{\lambda}\right)}+\frac{1}{1-f_{\lambda}^{\prime}\left(\widetilde{\xi}_{\lambda}\right)}=-1
$$

Hence $f_{\lambda}^{\prime}\left(\widetilde{\xi}_{\lambda}\right)$ tends to $(9+\sqrt{5}) / 4>1$, so $\widetilde{\xi}_{\lambda}$ cannot be attracting either. Therefore, $\lambda_{2} \notin \mathrm{cl} U$.

2. The existence of $\gamma_{0}$ and $\gamma_{\infty}$. We use the results concerning parabolic bifurcation proved in [DH1]. The following theorem (Theorem 1 from Lecture XI.2) was shown with the use of the "tour de valse" method. 


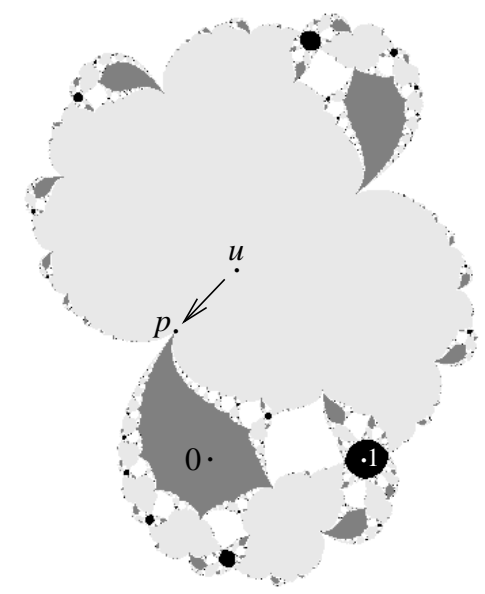

The parabolic map: $u$ tends to $p$
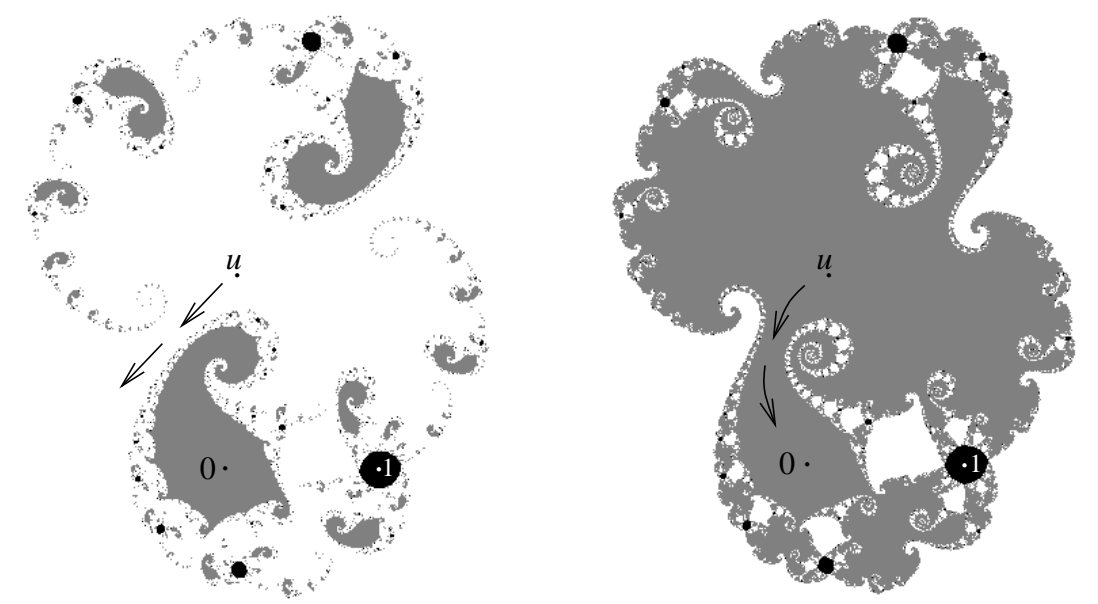

$u$ is in the exotic basin of $\infty$ (white)

$u$ is in the non-exotic basin of 0 (gray)

Fig. 8. Bifurcation of the Julia sets

Theorem 4.21 (Douady-Sentenac). Let $\Lambda$ and $V$ be some neighbourhoods of 0 in $\mathbb{C}$ and let $(\lambda, z) \mapsto g_{\lambda}(z)$ be a holomorphic map of $\Lambda \times V$ into $\mathbb{C}$. Suppose that $g_{\lambda}(0)=0$ for all $\lambda \in \Lambda, g_{0}^{\prime}(0)=\varrho_{0}=e^{2 i \pi p / q}, p / q \in \mathbb{Q}$ and the map $\lambda \mapsto \varrho(\lambda)=g_{\lambda}^{\prime}(0)$ is not constant. Suppose also that $g_{0}^{q}$ has the form $z \mapsto z+b_{0} z^{q+1}+\mathcal{O}\left(z^{q+2}\right)$ with $b_{0} \neq 0$. Let $L_{+}$and $L_{-}$be consecutive repelling and attracting axes for $g^{q}$ at 0 (i.e. the angle between $L_{+}$and $L_{-}$is equal to $2 \pi /(2 q))$. Denote by $S_{+}$(resp. $\left.S_{-}\right)$the open angle sector symmetric with respect to $L_{+}$(resp. $\left.L_{-}\right)$and forming an angle of measure $2 \pi /(4 q)$. 
Then there exists a disc $\Delta$ centred at 0 such that for every compact set $Q_{+}$ in $\Delta \cap S_{+}$, every compact set $Q_{-}$in $\Delta \cap S_{-}$and every neighbourhood $W$ of 0 in $\Lambda$ there exists $N_{0}$ such that for any continuous functions $\delta_{+}: W \rightarrow Q_{+}$ and $\delta_{-}: W \rightarrow Q_{-}$and for every $n \geq N_{0}$ there exists $\lambda \in W$ such that

$$
g_{\lambda}^{n q}\left(\delta_{-}(\lambda)\right)=\delta_{+}(\lambda)
$$

REMARK. The proof of the above theorem also gives:

(a) For every $j \leq[n / 2]$ we have $g_{\lambda}^{j q}\left(Q_{-}\right), g_{\lambda}^{-j q}\left(Q_{+}\right) \subset V$.

(b) The parameter $\lambda$ which exists according to the theorem tends to 0 as $n$ tends to infinity (uniformly with respect to $\delta_{+}, \delta_{-}$).

In our situation $q=1$ and $g_{\lambda}=f_{\lambda}$. Take $\lambda_{P}$ such that $P=f_{\lambda_{P}}$ and let $p$ be a fixed point of $f_{\lambda_{P}}$ with multiplier 1. Consider $z \in V$ for a small neighbourhood $V$ of $p$ and $\lambda \in W$ for a small neighbourhood $W$ of $\lambda_{P}$. By the square root of a holomorphic change of coordinates, we can assume $f_{\lambda}(p)=p$ for $\lambda \in W$. (It is obvious that in Theorem 4.21 we can replace the fixed point 0 by $p$ and the parameter $\lambda=0$ by $\lambda=\lambda_{P}$.) Moreover, $f_{\lambda_{P}}(z)=z+b_{0}(z-p)^{2}+\mathcal{O}\left((z-p)^{3}\right)$ with $b_{0} \neq 0$, because there is only one attracting petal since there is only one "free" critical point $u_{\lambda_{P}}$. Note that the parabolic map $P$ has the same combinatorics of internal rays in $B(0)$, $B(\infty)$ and $B(1)$ as $N$ (see Fig. 6).

Take $\zeta=0, \infty$ and $S_{+}, S_{-}, \Delta$ from the above theorem (here $\Delta$ is centred at $p$ ). By the local study of the parabolic map $f_{\lambda_{P}}$, the forward trajectory of any point eventually approaching $p$ is contained in a cusp containing $L_{-}$ with asymptotic behaviour like $1 / n$. Since $f_{\lambda_{P}}^{n}\left(u_{\lambda_{P}}\right) \rightarrow p$, we can take $n_{1}$ so large that

$$
\frac{\left|f_{\lambda_{P}}^{n_{1}+1}\left(u_{\lambda_{P}}\right)-f_{\lambda_{P}}^{n_{1}}\left(u_{\lambda_{P}}\right)\right|}{\operatorname{dist}\left(f_{\lambda_{P}}^{n_{1}}\left(u_{\lambda_{P}}\right), \partial S_{-}\right)}<\varepsilon_{1}
$$

for a small $\varepsilon_{1}$. Connect $f_{\lambda_{P}}^{n_{1}}\left(u_{\lambda_{P}}\right)$ to $f_{\lambda_{P}}^{n_{1}+1}\left(u_{\lambda_{P}}\right)$ by a straight line segment $I$ and let $U=\{z: \operatorname{dist}(z, I)<a \operatorname{diam} I\}$ for a small fixed $a$ such that $f_{\lambda_{P}}^{n_{1}-1}\left(u_{\lambda_{P}}\right) \notin \operatorname{cl} U$. Then there exists an inverse branch $F_{\lambda_{P}}$ of $f_{\lambda_{P}}^{-\left(n_{1}-1\right)}$ defined on $U$ such that $F_{\lambda_{P}}(U) \ni f_{\lambda_{P}}\left(u_{\lambda_{P}}\right), f_{\lambda_{P}}^{2}\left(u_{\lambda_{P}}\right)$. To construct such a branch, it is sufficient to take the branch of $f_{\lambda_{P}}^{-1}$ defined on the immediate basin of attraction $B(p)$ near $p$ and fixing $p$, and iterate this branch until we meet $f_{\lambda_{P}}\left(u_{\lambda_{P}}\right)$.

Hence for a sufficiently small neighbourhood $W$ of $\lambda_{P}$, for every $\lambda \in W$ there exists a branch $F_{\lambda}$ of $f_{\lambda}^{-\left(n_{1}-1\right)}$ on $U$ such that $F_{\lambda}(U) \ni f_{\lambda}\left(u_{\lambda}\right), f_{\lambda}^{2}\left(u_{\lambda}\right)$. Take a closed geometric disc $Q_{-}$centred at $f_{\lambda_{P}}^{n_{1}}\left(u_{\lambda_{P}}\right)$ of radius slightly less than $\operatorname{dist}\left(f_{\lambda_{P}}^{n_{1}}\left(u_{\lambda_{P}}\right), \partial S_{-}\right)$. Then $Q_{-} \subset S_{-} \cap \Delta$ and $U$ is contained in a disc with the same centre as $Q_{-}$and diameter less than $\varepsilon_{2} \operatorname{diam} Q_{-}$for a small $\varepsilon_{2}$. 
Let $\Phi_{\lambda}$ be the Böttcher coordinates on the maximal subset of $B(\zeta)$. Note that for the map $f_{\lambda_{P}}, \Phi_{\lambda_{P}}$ is defined on the entire $B(\zeta)$ and the curve $\Phi_{\lambda_{P}}^{-1}([0,1])$ is tangent to $L_{+}$. Therefore, for a fixed $r_{0}<1$ close to 1 , we can take a closed geometric disc $Q_{+} \subset S_{+} \cap \Delta$ such that for every $\lambda \in W$, the curve $\gamma_{\lambda}=\Phi_{\lambda}^{-1}\left(\left[r_{0}^{4}, r_{0}\right]\right)$ is contained in a disc with the same centre as $Q_{+}$and diameter less than $\varepsilon_{3} \operatorname{diam} Q_{+}$for a small $\varepsilon_{3}$. Take $r \in\left[r_{0}^{2}, r_{0}\right]$ and define

$$
\delta_{-}(\lambda)=f_{\lambda}^{n_{1}}\left(u_{\lambda}\right), \quad \delta_{+}(\lambda)=\Phi_{\lambda}^{-1}(r) .
$$

By definition, $\delta_{-}(W) \subset U \subset Q_{-}$and $\delta_{+}(W) \subset \bigcup_{\lambda \in W} \gamma_{\lambda} \subset Q_{-}$. By Theorem 4.21, there exists $N_{0}=N_{0}\left(W, n_{1}, r_{0}\right)$ such that for every $n$ greater than $N_{0}$ there exists $\lambda \in W$ such that

$$
f_{\lambda}^{n_{1}+n}\left(u_{\lambda}\right)=\Phi_{\lambda}^{-1}(r) .
$$

Consider $f_{\lambda}^{n-[n / 2]}$ on $Q_{-}$and $G_{\lambda}$ on $Q_{+}$for the inverse branch $G_{\lambda}$ of $f_{\lambda}^{-[n / 2]}$ specified by the forward trajectory of $u_{\lambda}$. Note that by Remark (a) after Theorem 4.21, $f_{\lambda}^{j}\left(Q_{-}\right), f_{\lambda}^{j}\left(G_{\lambda}\left(Q_{+}\right)\right) \subset V$ for $j=0, \ldots,[n / 2]$. Therefore, the distortion of $f_{\lambda}^{n-[n / 2]}$ on $U$ is bounded by $c\left(\varepsilon_{2}\right)$ and the distortion of $G_{\lambda}$ on $\gamma_{\lambda}$ is bounded by $c\left(\varepsilon_{3}\right)$, where $c\left(\varepsilon_{i}\right) \rightarrow 1$ as $\varepsilon_{i} \rightarrow 0$. Let $z_{1}=f_{\lambda}^{n_{1}+n-[n / 2]}\left(u_{\lambda}\right)$. By construction,

$$
z_{1} \in f_{\lambda}^{n-[n / 2]}(U) \cap G_{\lambda}\left(\gamma_{\lambda}\right) .
$$

This implies that for sufficiently small $\varepsilon_{i}, G_{\lambda}\left(\gamma_{\lambda}\right) \subset f_{\lambda}^{n-[n / 2]}\left(Q_{-}\right)$, so

$$
f_{\lambda}\left(z_{1}\right)=G_{\lambda}\left(\Phi_{\lambda}^{-1}\left(r^{2}\right)\right)=G_{\lambda}\left(f_{\lambda}\left(\Phi_{\lambda}^{-1}(r)\right)\right) .
$$

Let $J$ be the straight line segment connecting $z_{1}$ to $f_{\lambda}\left(z_{1}\right)$ and let $K=$ $\Phi_{\lambda}^{-1}\left(\left[r, r^{2}\right]\right)$. By bounded distortion,

$$
f_{\lambda}^{n-[n / 2]}(U) \supset\{z: \operatorname{dist}(z, J)<(a / 2) \operatorname{diam} J\} .
$$

Note that taking $r_{0}$ sufficiently close to 1 , we can assume that the length of $K$ is less than $q \operatorname{diam} K$ for a fixed $q>1$ arbitrarily close to 1 . When $\varepsilon_{3}$ is sufficiently small, the length of $G_{\lambda}(K)$ is less than $\widetilde{q} \operatorname{diam} G_{\lambda}(K)$ for $\widetilde{q}>1$ arbitrarily close to 1 . Since the ends of $G_{\lambda}(K)$ are the ends of $J$,

$$
G_{\lambda}(K) \subset f_{\lambda}^{n-[n / 2]}(U) .
$$

Consider the inverse branch of $f_{\lambda}^{-\left(n_{1}+n-1\right)}$ on $K$ defined as

$$
\left.F_{\lambda} \circ\left(f_{\lambda}^{n-[n / 2]} \mid\right)^{-1} \circ G_{\lambda}\right|_{K} .
$$

By construction, this branch goes along the forward trajectory of $f_{\lambda}\left(u_{\lambda}\right)$ and along the ray $\Phi_{\lambda}^{-1}((r, 1))$. Therefore, $f_{\lambda}\left(u_{\lambda}\right) \in B(\zeta)$ and

$$
\Phi_{\lambda}\left(f_{\lambda}\left(u_{\lambda}\right)\right)=\sqrt[2^{n_{1}+n-1}]{r} .
$$


By Corollary 4.5, $u_{\lambda} \in B(\zeta)$. Moreover,

$$
\Psi(\lambda)=\sqrt[2^{n_{1}+n-1}]{r}
$$

We have proved that there exist $N_{0}, r_{0}$ such that for every $r \in\left[r_{0}^{2}, r_{0}\right]$ and every $n \geq N_{0}$ there exists $\lambda=\lambda(r) \in W$ such that $\Psi(\lambda)=\sqrt[2^{n_{1}+n-1}]{r}$. Since $\sqrt[2 k]{r_{0}}=\sqrt[2]{2^{k+1}}$ for every $k$, there exists $r_{1}<1$ such that for every $r \in\left[r_{1}, 1\right)$ there exists $\lambda(r)$ such that $\Psi(\lambda)=r$. Moreover, by Remark (b) after Theorem 4.21, $\lambda(r) \rightarrow \lambda_{P}$ as $r \rightarrow 1^{-}$. Thus $\lambda_{P}$ is the landing point of the internal ray in the parameters $\mathcal{R}_{C}(0)$ for some component $C$ of $\Lambda_{\zeta}$. In this way we have proved the existence of the curves $\gamma_{0}, \gamma_{\infty}$.

3. $B(\infty)$ is exotic. By Corollary 4.5 , it is sufficient to check that $B(\infty)$ is not simply connected. As was proved in Subsection 4.4, for $\lambda=\lambda_{N}$ the set

$$
\operatorname{cl} R_{\infty}(0) \cup \operatorname{cl} R_{u}(0) \cup \operatorname{cl} R_{u}(1 / 2) \cup \operatorname{cl} R_{\infty}(1 / 2)
$$

is a Jordan curve separating 1 and $N(1)$ (see Fig. 6). We now show that for $\lambda$ near $\lambda_{P}$, if $f_{\lambda}\left(u_{\lambda}\right)$ lies on the ray $R_{\infty}(0)$, then there exists a Jordan curve in $B(\infty)$ separating 1 and $f_{\lambda}(1)$.

Let $A$ be a small open neighbourhood of $\operatorname{cl} R_{\infty}(0)$ for the map $f_{\lambda_{P}}$. Define

$$
E=A \cup \Delta \cup \bigcup_{i=0}^{n_{1}-1} f_{\lambda_{P}}^{i}\left(F_{\lambda_{P}}(U)\right)
$$

for $\Delta, n_{1}, F_{\lambda_{P}}$ and $U$ from Part 2 of the proof. By definition, $E$ is an open connected set containing $\infty, R_{\infty}(0)$, the parabolic fixed point $p$ and the critical value $f_{\lambda_{P}}\left(u_{\lambda_{P}}\right)$. Consider $f_{\lambda_{P}}^{-1}(E)$. It consists of two components: $E_{1}$ containing $R_{\infty}(0), R_{\infty}(1 / 2), u_{\lambda_{P}}$, and $E_{2}$ containing $\widetilde{R}_{\infty}(0)$, $f_{\lambda_{P}}^{-1}\left(f_{\lambda_{P}}\left(u_{\lambda_{P}}\right)\right) \backslash\left\{u_{\lambda_{P}}\right\}$. Moreover, cl $E_{1} \cap \mathrm{cl} E_{2}=\emptyset$. Take $\lambda$ close to $\lambda_{P}$ such that $f_{\lambda}\left(u_{\lambda}\right) \in R_{\infty}(0)$ and let $\Gamma$ be the part of $R_{\infty}(0)$ between $\infty$ and $f_{\lambda}\left(u_{\lambda}\right)$. By Part 2 of the proof, if $\lambda$ is sufficiently close to $\lambda_{P}$, then $\Gamma \subset E$. Hence $f_{\lambda}^{-1}(\Gamma)$ has two components: $\Gamma_{1} \subset E_{1}$ and $\Gamma_{2} \subset E_{2}$. By construction, $\Gamma_{1}$ is a Jordan curve in $B(\infty)$ separating 1 and $f_{\lambda}(1)$. Therefore $B(\infty)$ is not simply connected.

4. $B(0)$ is not exotic. To prove that $B(0)$ is simply connected it suffices to show that $\widehat{\mathbb{C}} \backslash B(0)$ is connected. As was proved in Subsection 4.4, for $\lambda=\lambda_{N}$ the set

$$
\begin{aligned}
\Gamma^{\prime}=\operatorname{cl} R_{\infty}(1 / 3) \cup \operatorname{cl} R_{\infty}(2 / 3) & \cup \operatorname{cl} R_{1}(0) \\
\cup & \operatorname{cl} R_{1}(1 / 2) \cup \operatorname{cl} \widetilde{R}_{\infty}(1 / 3) \cup \operatorname{cl} \widetilde{R}_{\infty}(2 / 3)
\end{aligned}
$$

is connected and joins $B(\infty), f_{\lambda_{N}}^{-1}(B(\infty)), B(1)$ and $f_{\lambda_{N}}^{-1}(B(1))$ (see Fig. 6). Note that $\Gamma^{\prime}$ consists of periodic rays and their preimages. It is easy to see that the parabolic map $f_{\lambda_{P}}$ has the same combinatorics of the rays in $B(\infty)$ 
and $B(1)$. Hence for $\lambda=\lambda_{P}$ the curve $\Gamma^{\prime}$ is still connected. The same holds after a small perturbation of $\lambda$ near $\lambda_{P}$ such that $u_{\lambda} \in B(0)$. Therefore the set

$$
B=\bigcup_{n \geq 0} f_{\lambda}^{-n}\left(B(\infty) \cup B(1) \cup \Gamma^{\prime}\right)
$$

is connected, so $\mathrm{cl} B$ is connected. But $\operatorname{cl} B=\widehat{\mathbb{C}} \backslash B(0)$. Hence $B(0)$ is simply connected.

REMARK. In Theorem 4.20 we assumed that for $N$ the set $\partial B(u) \cap \partial B(\infty)$ is infinite. Such a situation is presented in Fig. 6. Suppose now that for the map $N, \partial B(u) \cap \partial B(\infty)$ is finite (actually this means that $\partial B(u) \cap \partial B(\infty)$ consists of one point, see Subsection 4.4). In Fig. 6 this corresponds to the exchange of 0 and $u$. Then repeating the proof of Theorem 4.20 we show the existence of a similar parabolic bifurcation, but now neither $B(0)$ nor $B(\infty)$ is exotic.

Considering the parameter plane $\Lambda$ we conclude that starting from the Newton map $N_{1}$ we arrive at some parabolic parameter $\lambda_{P_{1}}$ which is the landing point of the parameter rays $\mathcal{R}_{-C_{E}}(0)$ and $\mathcal{R}_{C_{R 1}}(0)$, and starting from $N_{2}$ we come to $\lambda_{P_{2}}$ which is the landing point of $\mathcal{R}_{C_{R 1}}(0)$ and $\mathcal{R}_{-\bar{C}_{R 1}}(0)$. See Figs. 2 and 3.

\section{References}

[Ba] K. Barański, Connectedness of the basin of attraction for rational maps, Proc. Amer. Math. Soc. (6) 126 (1998), 1857-1866.

[BH] B. Branner and J. Hubbard, The iteration of cubic polynomials, II: Patterns and parapatterns, Acta Math. 169 (1992), 229-325.

[CGS] J. H. Curry, L. Garnett and D. Sullivan, On the iteration of a rational function: computer experiments with Newton's method, Comm. Math. Phys. 91 (1983), 267-277.

[DH1] A. Douady et J. H. Hubbard, Etude dynamique des polynômes complexes, I et II, avec la collaboration de P. Lavours, Tan Lei et P. Sentenac, Publication d'Orsay 84-02, 85-04, 1984-1985.

[DH2] - - - On the dynamics of polynomial-like mappings, Ann. Sci. École Norm. Sup. (4) 18 (1985), 287-343.

[HP] F. von Haeseler and H.-O. Peitgen, Newton's method and complex dynamical systems, Acta Appl. Math. 13 (1988), 3-58.

[He] J. Head, The combinatorics of Newton's method for cubic polynomials, Ph.D. thesis, Cornell University, Ithaca, 1987.

[MSS] R. Mañé, P. Sad and D. Sullivan, On the dynamics of rational maps, Ann. Sci. École Norm. Sup. (4) 16 (1983), 193-217.

[Mi1] J. Milnor, Dynamics in one complex variable: introductory lectures, preprint, SUNY at Stony Brook, IMS \# 1990/5.

[Mi2] -, Geometry and dynamics of quadratic rational maps, Experiment. Math. 2 (1993), 37-83. 
[P1] F. Przytycki, Iterations of rational functions: which hyperbolic components contain polynomials?, Fund. Math. 149 (1996), 95-118.

[P2] - Remarks on simple-connectedness of basins of sinks for iterations of rational maps, in: Banach Center Publ. 23, PWN, 1989, 229-235.

[Re1] M. Rees, A partial description of parameter space of rational maps of degree two, I: Acta Math. 168 (1992), 11-87; II: Proc. London Math. Soc. (3) 70 (1995), 644-690.

[Re2] - Components of degree two hyperbolic rational maps, Invent. Math. 100 (1990), 357-382.

[Ro] P. Roesch, Topologie locale des méthodes de Newton cubiques, Ph.D. thesis, École Norm. Sup. de Lyon, 1997.

[Se] G. Segal, The topology of spaces of rational functions, Acta Math. 143 (1979), 39-72.

[Sh] M. Shishikura, The connectivity of the Julia set of rational maps and fixed points, preprint, Inst. Hautes Études Sci., Bures-sur-Yvette, 1990.

[Ta] L. Tan, Branched coverings and cubic Newton maps, Fund. Math. 154 (1997), 207-260.

Institute of Mathematics

Warsaw University

Banacha 2

02-097 Warszawa, Poland

E-mail: baranski@mimuw.edu.pl

Received 1 December 1997;

in revised form 7 July 1998 